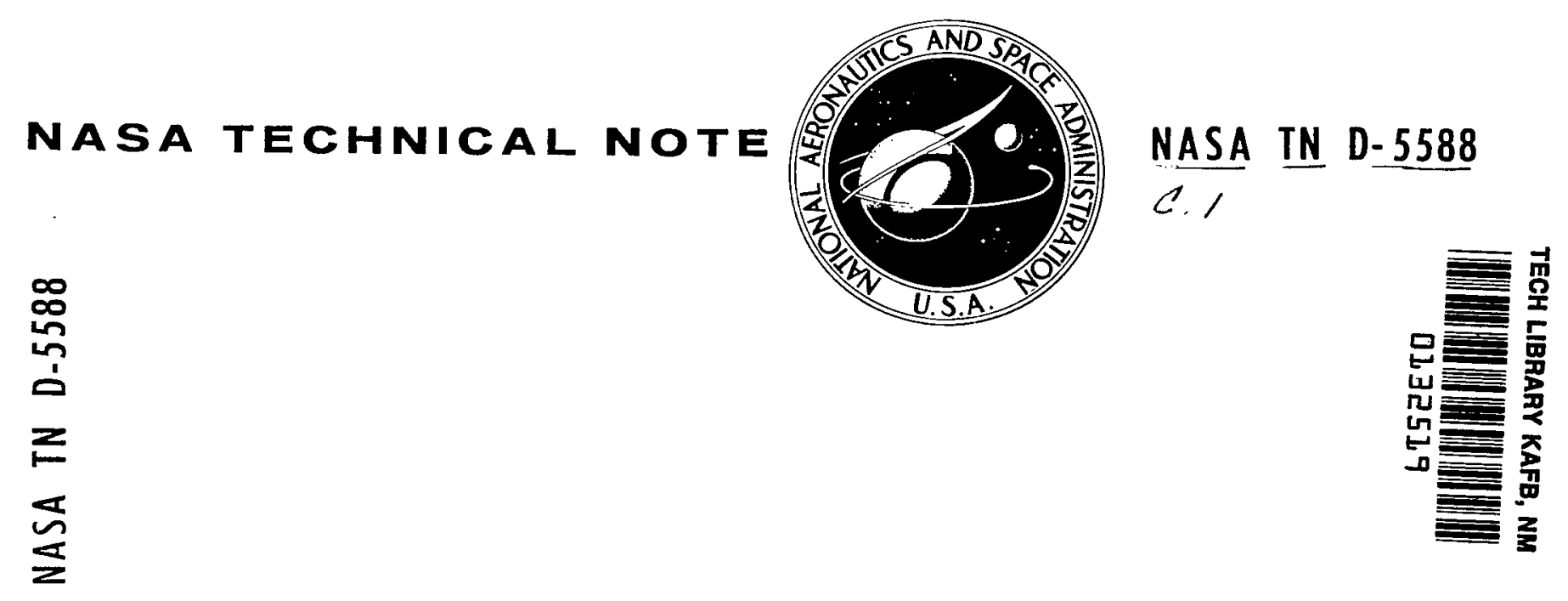

IOAN COPY : RETURH TO AFWL (WLOL)

KIRTIAND AFB, N MEX

\title{
THEORY OF GALVANOMAGNETIC EFFECTS IN METALS
}

by Gale Fair

Lewis Research Center

Cleveland, Obio

NATIONAL aERONAUtics AND SPACE ADMINISTRATION - WASHINGTON, D. C. - DECEMBER 1969 
1. Report No NASA TN D-5588

2. Government Accession No.

4. Title and Subtitle THEORY OF GALVANOMAGNETIC EFFECTS IN METALS

7. Author(s)

Gale Fair

9. Performing Organization Name and Address Lewis Research Center

National Aeronautics and Space Administration Cleveland, Ohio 44135

12. Sponsoring Agency Name and Address

National Aeronautics and Space Administration Washington, D.C. 20546

15. Supplementary Notes

16. Abstract

A solution to the linearized Boltzmann equation has been found that describes the galvanomagnetic properties of metals. The use of a vector mean-free-path function results in a solution for the conductivity tensor that is valid for any strength and orientation of the applied magnetic field. A discussion is given of the implementation of this theory to give a quantitative description of the galvanomagnetic properties of a metal with a specified Fermi surface and with a model for the scattering of the conduction electrons. Finally, two scattering models are treated for a metal having a spherical Fermi surface. The familiar result for a free-electron gas is derived, and the case of a degenerate scattering kernel is treated in detail.
17. Key Words (Suggested by Author(s)) Boltzmann equation Electric conductivity Galvanomagnetism Magnetor esistance Fermi crystal Metal crystal

19. Security Classif. (of this report) Unclassified For sale by the Clearingh

* For sale by the Clearinghouse for Federal Scientific and Technical Information
18. Distribution Stotement

Unclassified - unlimited tegral equation Group theory
Fredholm in20. Security Classif. (of this page)
Unclassified
21. No. of Pages

59
22. Price* $\$ 3.00$ Springfield, Virginia 22151 


\section{CONTENTS}

Page

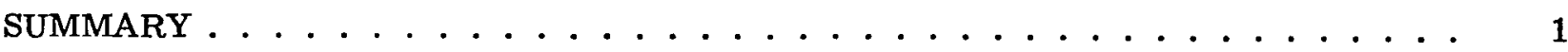

THE BOLTZMANN EQUATION . . . . . . . . . . . . . . . . . . 1

SOLUTION OF THE BOLTZMANN EQUATION . . . . . . . . . . . . . . . 11

SYMMETRY PROPERTIES OF THE SCATTERING EIGENFUNCTIONS . . . . . . . 19

STRUCTURE OF THE CALCULATIONS . . . . . . . . . . . . . . . . . 26

AN EXAMPLE: SPHERICAL FERMI SURFACE AND

ISOTROPIC SCATTERING . . . . . . . . . . . . . . . . . . . 30

DEGENERATE KERNEL THEORY . . . . . . . . . . . . . . . . . 36

CONCLUSIONS . . . . . . . . . . . . . . . . . . . . . . 42

APPENDIXES

A - SCATTERING AND MAGNETIC SCATTERING

EIGENVALUE PROBLEM . . . . . . . . . . . . . . . . . . . . . 44

B - PROOF OF GROUP THEORETIC THEOREMS AND RESULTS . . . . . . . . 48

REFERENCES . . . . . . . . . . . . . . . . . . . . . . 54 


\title{
THEORY OF GALVANOMAGNETIC EFFECTS IN METALS
}

\author{
by Gale Fair
}

\section{Lewis Research Center}

\section{SUMMARY}

The Boltzmann equation for the distribution function of conduction electrons in a metal is used to describe the response of the electronic system to applied magnetic and electric fields. The vector mean-free-path formulation, originally developed by Taylor to calculate the zero-magnetic-field resistance of metals, is used to find an exact solution to the linearized Boltzmann equation for static and homogeneous applied fields. The conductivity tensor is evaluated in terms of two operators, one describing the scattering between electronic states and the second including the effect of the Lorentz force. The eigenfunctions of these operators provide a natural framework for a quantitative description of the galvanomagnetic properties of a metal, for both an assumed Fermi surface and a scattering model for the conduction electrons.

Of particular importance is the treatment of the electronic scattering processes, a treatment sufficiently complete so as to allow a separation of effects due to the scattering processes from the effects arising from topological features of the Fermi surface. To demonstrate the implementation of the theory, and to illustrate the application of the representation theory of finite groups, two examples are considered. The free-electron gas, with spherically symmetric scattering, is shown to give the result familiar from a relaxation-time approximation (i.e., a Hall effect but no magnetoresistance). The second example is a treatment of a degenerate scattering kernel, still within the framework of a spherical Fermi surface. A special case of this degenerate kernel theory is given in a recent work by Jones and Sondheimer, and a sample calculation is presented for comparison with this work.

\section{THE BOLTZMANN EQUATION}

A galvanomagnetic experiment measures the voltage response to an externally applied current for a metal sample in a magnetic field. The experimentally determined 
quantity is the resistivity tensor of the metal as a function of the magnetic field (both strength and orientation) and the temperature. The experiment constitutes a probe of the properties of the conduction electrons in the metal and should, in principle, yield information about the scattering processes present and the structure of the Fermi surface. Although galvanomagnetic experiments have been performed for many years, no general theory has yet appeared that gives a quantitative connection with experiment. In this report a semiclassical theory is presented for the galvanomagnetic effects in metals within the framework of the independent-particle description and the Boltzmann equation. A closed-form solution of the Boltzmann equation is found that is valid for any strength of the magnetic field, and the resulting conductivity tensor is calculated. After a discussion of the implementation of the formalism for calculations, consideration is given to the special case of a metal having a spherical Fermi surface, for two different scattering models.

The semiclassical theory for electrons in metals is based upon the existence of a distribution function $f(\vec{r}, \bar{k}, t)$. The quantity $f \overrightarrow{(r}, \vec{k}, t) \overrightarrow{d r} \overline{d k}$ is the probability of finding

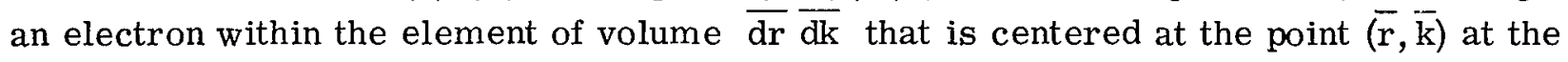
time $t$ in the six-dimensional space of position $\bar{r}$ and Bloch wave-number index $\bar{k}$ (ref. 1). For a spatially homogeneous metallic sample with no thermal gradients present, it is customary to assume that the spatial variation of $f$ is negligible ( $r$ ef. 2). Then for $f$ we can write $f(\bar{k}, t)$, or $f_{k}(t)$. For a system of electrons in equilibrium, the Pauli exclusion principle determines the distribution of the electrons in momentum space. The distribution function depends only upon the energy of the electron and is the Fermi function, $f^{0}\left(\epsilon_{k}\right)=\left\{1+\exp \left[\left(\epsilon_{k}-\zeta\right) / k_{B} T\right]\right\}^{-1}$; where $\epsilon_{k}$ is the energy of the electron, $\mathrm{T}$ is the temperature of the system, $\zeta$ is the chemical potential, and $\mathrm{k}_{\mathrm{B}}$ is the Boltzmann constant. This function implies a discontinuity in the energy distribution of the electrons when the system is at absolute zero. When $\mathrm{T}=0, \mathrm{f}^{0}\left(\epsilon_{\mathrm{k}}\right)$ is 1 if $\epsilon_{\mathrm{k}}<\zeta$ and zero if $\epsilon_{\mathrm{k}}>\zeta$. The value of the chemical potential at absolute zero $\zeta(\mathrm{T}=0)$ defines the parameter $\epsilon_{F}$, the Fermi energy. For the special case of a system of free electrons, the occupied states in wave-number space form a sphere, of radius $\mathrm{k}_{\mathrm{F}}=\sqrt{2 \mathrm{~m} \epsilon_{\mathrm{F}}} / \mathrm{h}$, known as the Fermi sphere.

The existence of a discontinuity in the distribution function as a function of wave vector $\overline{\mathrm{k}}$ is very useful for the discussion of the transport properties of metals. The locus of this discontinuity in $\mathrm{k}$-space at $\mathrm{T}=0$ is called the Fermi surface. Although the Fermi surface for the system of conduction electrons in a metal is distorted from a sphere by the presence of the lattice of ion cores, experimental evidence (ref. 3) shows the existence of such a discontinuity surface at low temperatures. The galvanomagnetic experiment is one of a class of experiments designed to probe the Fermi surface of metals (refs. 3 and 4). As will be seen later, it is the Fermi surface that helps to determine the $\mathrm{k}$-states that may participate in transport effects. 
Before the Boltzmann equation is presented, it is useful to briefly summarize the semiclassical view of a conduction electron in a metal. At a finite temperature, the discontinuity in the distribution function expressed in terms of $\epsilon_{\mathrm{k}}$ is not sharp, the energy range which separates the totally occupied states from the totally unoccupied states being a band of width approximately $\mathrm{k}_{\mathrm{B}} \mathrm{T}$. Since this thermal broadening constitutes a range of energy which is small compared with the Fermi energy, there is still an experimentally well-defined Fermi surface, whose detailed shape is determined by the number of valence electrons available, the symmetry of the ionic lattice, and the interactions between the electrons and their environment (ref. 3). An electron in a state $\overline{\mathrm{k}}$ at the Fermi surface moves with the velocity $\nabla_{\mathbf{k}} \in \overline{(\mathrm{k})} / \hbar$, which is, in fact, the group velocity of the wave packet describing the electron (ref. 5). An electron in a state $\overline{\mathbf{k}}$ may undergo a scattering, which is pictured as a discontinuous change from the initial state $\overrightarrow{\mathrm{k}}$ to a final state $\overline{\mathrm{k}}^{\prime}$. The final state may or may not have the same energy as the initial state, depending upon the nature of the scattering process. Common causes of scattering (ref. 2) are impurities, physical defects in the crystal such as point defects or dislocations, and, at a finite temperature, the thermal vibrations of the lattice (i. e., the phonons). A satisfactory theory of the transport properties of a metal must be capable of describing the response of the electronic system to external forces, and include specifically the effects due to the scattering processes and the nature of the Fermi surface.

The semiclassical description of transport phenomena is governed by the Boltzmann equation ( $\mathrm{BE}$ ) for the electronic distribution function. The $\mathrm{BE}$ is the equation of motion for the distribution and is obtained by a consideration of the processes which determine the net change in occupation of the volume element $\overline{\mathrm{dr}} \overline{\mathrm{dk}}$. Although the system is not in an equilibrium state when external electric and magnetic fields are present, it is customary to assume steady-state conditions. This means that there is no net flow from the volume element under observation, and thus the distribution function is not a function of time. The effect of the external forces must balance the rate of change of occupation caused by the scattering. The equation of continuity, applied to the six-dimensional phase space volume element, gives

$$
\frac{\partial f_{k}}{\partial t}=-\nabla_{r_{k}} \cdot \frac{d \bar{r}}{d t}-\nabla_{k^{f}} f \cdot \frac{d \bar{k}}{d t}-\left(\frac{\partial f}{\partial t}\right)_{c o l l}=0
$$

where the subscripts on the gradients indicate the variables of the differentiation and the term $(\partial f / \partial t)_{\text {coll }}$ is symbolic for the rate of change in $f$ caused by scattering. In a galvanomagnetic experiment the temperature is uniform throughout the sample. In this case (ref. 2), the spatial derivative of $f_{k}$ will vanish. For a Bloch electron in electric and magnetic fields, the time rate of change of the crystal momentum is given by the 
L.: Th:

$$
\hbar \frac{\mathrm{d} \overline{\mathrm{k}}}{\mathrm{dt}}=\mathrm{e}\left(\overline{\mathrm{E}}+\frac{1}{\mathrm{c}} \overline{\mathrm{v}}_{\mathrm{k}} \times \overline{\mathrm{H}}\right)
$$

The scattering term is obtained by considering the balance between scattering into ans rrom the state $\vec{k}$. Let $Q\left(\bar{k}, \bar{k}^{\prime}\right)$ represent the transition probability per unit time for $s=\operatorname{serins}$ from the initial occupied state $\overline{\mathrm{k}}$ to the final empty state $\overline{\mathrm{k}}^{\prime}$. The occupation $\therefore$ i.:e initial and final states is restricted by the Pauli exclusion principle for the electrons. and so the scattering rate must include factors to ensure that the initial state is accupied while the final state is initially unoccupied. The net change in the occupation of the state $\overline{\mathrm{k}}$ due to scattering is (ref. 7)

$$
\left(\frac{\partial \mathrm{f}}{\partial \mathrm{t}}\right)_{\text {coll }}=\int\left\{\mathrm{Q}\left(\overline{\mathrm{k}^{\prime}}, \overline{\mathrm{k}}\right) \mathrm{f}\left(\overline{\mathrm{k}^{\prime}}\right)[1-\mathrm{f}(\overline{\mathrm{k}})]-\mathrm{Q}\left(\overline{\mathrm{k}}, \overline{\mathrm{k}^{\prime}}\right) \mathrm{f}(\overline{\mathrm{k}})\left[1-\mathrm{f}\left(\overline{\mathrm{k}^{\prime}}\right)\right]\right\} \overline{\mathrm{d}} \mathrm{k}^{\prime}
$$

The volume element in the integral of equation (3) is defined as (ref. 2)

$$
\overline{\mathrm{dk}}=\frac{\mathrm{V}}{(2 \pi)^{3}} \mathrm{~d}^{3} \mathrm{k}
$$

where $V$ is the volume of the sample. The transition probability per unit time $Q\left(\overline{\mathrm{k}}, \overline{\mathrm{k}}^{\prime}\right)$ provides the connection with the microscopic description of the electronic properties of metals. This probability of scattering from a state $\overline{\mathrm{k}}$ to a state $\overline{\mathrm{k}}^{\prime}$ is proportional to the square of the matrix element of the transition matrix for the scattering between the initial and final states (ref. 2).

The form of the scattering integral (eq. (3)) may be simplified by the assumption that the scattering is elastic. Under this assumption the transition probability is symmetric (ref. 2), that is,

$$
\mathrm{Q}\left(\overline{\mathrm{k}}, \overline{\mathrm{k}}{ }^{\prime}\right)=\mathrm{Q}(\overline{\mathrm{k}}, \overline{\mathrm{k}})
$$

where $\mathrm{Q}$ includes a factor $\delta\left(\epsilon_{\mathrm{k}}-\epsilon_{\mathrm{k}^{*}}\right)$ to conserve energy between the initial and final states. This simplification gives from equation (3),

$$
\left(\frac{\partial \mathrm{f}}{\partial \mathrm{t}}\right)_{\text {coll }}=\int \mathrm{Q}\left(\overline{\mathrm{k}}, \overline{\mathrm{k}^{\prime}}\right)\left[\mathrm{f}\left(\overline{\mathrm{k}^{\prime}}\right)-\mathrm{f}(\overline{\mathrm{k}})\right] \overline{\mathrm{d} \mathrm{k}^{\prime}}
$$


And the BE for elastic scattering is

$$
\frac{\mathrm{e}}{\hbar}\left(\overline{\mathrm{E}}+\frac{1}{\mathrm{c}} \overrightarrow{\mathrm{v}}_{\mathrm{k}} \times \overline{\mathrm{H}}\right) \cdot \nabla_{\mathrm{k}^{\mathrm{f}}} \mathrm{f}(\overline{\mathrm{k}})=\int \mathrm{Q}\left(\overline{\mathrm{k}}, \overline{\mathrm{k}^{\prime}}\right)\left[\mathrm{f}(\overline{\mathrm{k}})-\mathrm{f}\left(\overline{\mathrm{k}^{\prime}}\right)\right] \overline{\mathrm{dk}}^{\prime}
$$

with the energy-conserving $\delta$-function implicit in $Q\left(\overrightarrow{\mathrm{k}}, \overline{\mathrm{k}}^{\prime}\right)$.

There are two questions that have been passed over in the derivation of the $\mathrm{BE}$ (eq. (5)). The first question is the effect of the omission of inelastic scattering processes in passing from equation (3) to (4). The second is concerned with the meaning of the integral in equation (4). The symmetry of the transition probability holds true only when the scattering is elastic, as assumed. A more general relation may be derived from the observation that in the equilibrium state, in which the distribution function is the Fermi function, the integral of equation (3) must vanish, so that

$$
\mathrm{Q}\left(\overline{\mathrm{k}}, \overline{\mathrm{k}}^{\prime}\right) \mathrm{f}^{0}\left(\epsilon_{\mathrm{k}}\right)\left[1-\mathrm{f}^{0}\left(\epsilon_{\mathrm{k}^{\prime}}\right)\right]=\mathrm{Q}\left(\overline{\mathrm{k}^{\prime}}, \overline{\mathrm{k}}\right) \mathrm{f}^{0}\left(\epsilon_{\mathrm{k}^{\prime}}\right)\left[1-\mathrm{f}^{0}\left(\epsilon_{\mathrm{k}}\right)\right]
$$

Thus, even though $Q$ is not symmetric, there is a function (ref. 7)

$$
P\left(\bar{k}, \bar{k}^{\prime}\right) \equiv Q\left(\bar{k}, \bar{k}^{\prime}\right) f^{0}\left(\epsilon_{k^{\prime}}\right)\left[1-f^{0}\left(\epsilon_{k^{\prime}}\right)\right]=P\left(\overline{k^{\prime}}, \bar{k}\right)
$$

that has the desired symmetry. In terms of this function, the time rate of change of $\mathbf{f}_{k}$ due to arbitrary scattering processes is

$$
\left(\frac{\partial \mathrm{f}}{\partial \mathrm{t}}\right)_{\text {coll }}=\int \mathrm{P}\left(\overline{\mathrm{k}}, \overline{\mathrm{k}}^{\prime}\right)\left\{\begin{array}{c}
\mathrm{f}\left(\overline{\left.\mathrm{k}^{\prime}\right)}[1-\mathrm{f} \overline{\mathrm{k}})\right] \\
\mathrm{f}^{0}\left(\epsilon_{\mathrm{k}^{\prime}}\right)\left[1-\mathrm{f}^{0}\left(\epsilon_{\mathrm{k}}\right)\right]
\end{array}-\frac{\mathrm{if} \overline{(\bar{k})}\left[1-\mathrm{f}\left(\overline{\mathrm{k}^{\prime}}\right)\right]}{\mathrm{f}^{0}\left(\epsilon_{\mathrm{k}}\right)\left[1-\mathrm{f}^{0}\left(\epsilon_{\mathrm{k}^{\prime}}\right)\right]}\right\} \mathrm{dk}^{\prime}
$$

In principle, the formalism which is presented in this report may be carried through by using this more general form for the scattering term. This, in fact, was the approach followed by Taylor (refs. 8 and 9 ) in his solution of the zero-magnetic-field BE. Inelastic electron-phonon scattering makes a negligible contribution to galvanomagnetic effects at the low temperatures at which these experiments are performed (ref. 7). The inclusion of only elastic scattering in the present work should still provide a useful comparison with experiment.

The problem involved in defining the range of the $\overline{\mathrm{k}}$-space integral of equation (4) is a question of nonuniqueness commonly encountered in the band theory of metals. This nonuniqueness has its origins in the translational symmetry of the lattice. In a pure metal crystal of infinite extent there are a countably infinite number of points in the metal which are equivalent. These points are connected by finite displacement vectors, 
not by infinitesimal transformations. A translational invariance in configuration space always has implications in the momentum properties of a quantum-mechanical system (ref. 10). In band theory this gives rise to the definition of the Brillouin zone and the use of two pictures, the extended zone scheme and the reduced zone scheme. The extended zone scheme permits all values of $\overline{\mathbf{k}}$; equivalent points on the multiply occurring Fermi surfaces are connected by reciprocal lattice vectors. In the reduced zone scheme a Brillouin zone is constructed about a selected lattice point in $\mathrm{k}$-space, the Brillouin zone being the locus of points nearer this selected lattice point than any other. In this case the only scattering transitions allowed are those which connect two points on this single Fermi surface (which, of course, may be composed of a number of separate pieces). A much clearer description of the reduced and extended zone schemes, along with a third, the periodic zone scheme, is given by Harrison (ref. 11).

These different ways of picturing the wave-number distribution of the electrons must give identical results for the electronic properties of the system, and the choice of which scheme to use is dictated primarily by considerations of convenience and clarity. The convention adopted in this report is that integrals over wave-number space (which will ultimately become integrals over the Fermi surface) are considered to be over a single Brillouin zone. Whenever it becomes necessary to explicitly define ' $'$ ' ' region of integration, therefore, the reduced zone scheme shall be used.

The most fruitful attempts to describe the transport processes in metals by means of the BE have started with the linearization of equation (5). The distribution function $f(\bar{k})$ is expanded in a power series in the electric field, and all terms beyond the first power of $\bar{E}$ are dropped. The deviation from equilibrium then is written as (ref. 7 )

$$
f(\bar{k})-f^{0}\left(\epsilon_{k}\right)=g(\bar{k}) \frac{\partial f^{0}}{\partial \epsilon_{k}}
$$

The linearization results in the following form for the $\mathrm{BE}$ :

$$
\mathrm{e} \overline{\mathrm{E}} \cdot \overline{\mathrm{v}}_{\mathrm{k}}+\frac{\mathrm{e}}{\hbar \mathrm{c}} \overline{\mathrm{v}}_{\mathrm{k}} \times \overline{\mathrm{H}}^{\mathrm{k}} \cdot \nabla_{\mathrm{k}} \mathrm{g}(\overline{\mathrm{k}})=\int \mathrm{Q}\left(\overline{\mathrm{k}}, \overline{\mathrm{k}}^{\prime}\right)\left[\mathrm{g}(\overline{\mathrm{k}})-\mathrm{g}\left(\overline{\mathrm{k}}^{\prime}\right)\right] \overline{\mathrm{dk}} \overline{\mathrm{k}}^{\prime}
$$

Most solutions of this equation have started with the assumption that the scattering term may be simplified by means of a relaxation-time approximation. This approximation was used by R. G. Chambers (ref. 12), for example, who showed that there exists an expression for the deviation of the distribution function from equilibrium if the scattering is governed by a relaxation time, that is, if the probability of a scattering occurring in a time $\mathrm{dt}$ is proportional to $\mathrm{dt}$ with the proportionality constant $1 / \tau(\overline{\mathrm{k}})$. This is equivalent to the identification 


$$
\left(\frac{\partial \mathrm{f}}{\partial \mathrm{t}}\right)_{\mathrm{coll}}=-\frac{\left[\mathrm{f}(\overline{\mathrm{k}})-\mathrm{f}^{0}\left(\epsilon_{\mathrm{k}}\right)\right]}{\tau(\overline{\mathrm{k}})}
$$

Budd (ref. 13) has shown that Chambers' solution to the BE (which was derived originally to include only an electric field) needed only slight modification to include a magnetic field, and that the result was an exact solution to the linearized, relaxation-time BE. The resulting solution is in terms of integrals over the past history of the electron, and its exact form is not of interest here. Although the form of the solution makes it difficult to apply easily to the properties of real metals, applications have been made to the theory of transport properties of semiconductors (ref. 14), the attenuation of ultrasonic waves in metals (ref. 15), and the asymptotic form of the magnetoconductivity tensor for metals (e.g., refs. 16 and 17).

The introduction of a relaxation time for the scattering is primarily a device for reducing the $\mathrm{BE}$ (in the form of eq. (9)) to a differential equation for the distribution function. The applied magnetic field drives a conduction electron in an orbit around the Fermi surface in a plane perpendicular to the direction of the applied field. Such a simplification of the BE has a natural set of coordinates in terms of energy, relative position on an orbit, and the position of the orbit on the surface. This description allows the contribution of an electron to the transport current to be interpreted in terms of the details of the Fermi surface sampled in its trajectory. Thus, there is a natural framework available to consider the effects of closed orbits, orbits that are closed but extend through many zones (in the extended zone scheme), or even trajectories that extend indefinitely through k-space, as in the case of copper. The effect of each of these types of trajectories may be considered as a function of the lifetime of an electron in its orbit before a scattering occurs.

This approach to the transport problem was carried far by the work of Lifshitz, Azbel', and Kaganov (ref. 18) and Lifshitz and Peschanskii (refs. 19 and 20). These authors cataloged a large number of possible Fermi surface shapes in terms of their contributions to the transport properties in high and low magnetic field strengths. This catalog of qualitative descriptions still provides the working tools for the experimentalist who is investigating the shape of a Fermi surface from galvanomagnetic experiments.

The preceding discussion was concerned with work which sacrificed any attempt at a detailed description of the scattering, the result being a problem which is mathematically simple. Although the usefulness of the results, in terms of understanding the effects of Fermi surface topology on transport properties, justified the relaxation-time approximation, this approach is not adequate to support current experimental work. The task of measuring Fermi surface dimensions has been taken over by experiments relying upon other phenomena, such as the de Haas - van Alphen effect. The galvanomagnetic ef- 
fects, on the other hand, are more useful for investigations of the scattering of electrons in metals. Clearly, the relaxation-time approximation is not adequate for such investigations, and more general theories must be developed.

One approach to a more satisfactory treatment of the scattering term in the BE was introduced by Sondheimer (ref. 21). He made the ad hoc assumption that the scattering transition probability could be written as a finite sum of products of a function of $\overline{\mathrm{k}}$ with a function of $\overline{\mathrm{k}}^{\prime}$, that is,

$$
Q\left(\bar{k}, \bar{k}^{\prime}\right)=\sum_{j=1}^{N} b_{i j} q_{i}(\bar{k}) q_{j}\left(\overline{k^{\prime}}\right) \delta\left(\epsilon_{k}-\epsilon_{k^{\prime}}\right)
$$

The matrix $b_{i j}$ is real and symmetric, and the real functions $a_{i}(\bar{k})$ are arbitrary, except that they must be square-integrable in the Brillouin zone. In the theory of Fredholm integral equations, such a kernel is called degenerate, and an extensive theory of such integral equations has been developed (Mikhlin (ref. 22) and Courant and Hilbert (ref. 23)). The effect of this replacement (eq. (11)) in a Fredholm equation is that the integral equation is replaced by a finite set of linear equations for the matrix elements $b_{i j}$, if the functions $q_{i}$ are chosen properly. The effect of this assumption on the BE is to reduce equation (9) to a finite set of coupled differential equations. With this reduction, Sondheimer (ref. 21) and Jones and Sondheimer (ref. 24) were able to discuss the resistivity and asymptotic longitudinal magnetoresistance of metals with anisotropic properties. Although the use of the degenerate kernel was an arbitrary construction, a partial justification of this form for some types of scattering has been made by Mann (refs. 25 to 27). A further discussion of this theory and its relation to the present work is made in the section DEGENERATE KERNEL THEORY.

The approaches to the transport problem discussed here have had one feature in common - the linearization assumption of equation (8) or its equivalent. This linearization assumes that the deviation from equilibrium of the distribution function is proportional to the magnitude of the electric field. The starting point for the present work, on the other hand, is the observation by Taylor (refs. 8 and 9) that as a consequence of the linearization assumption equation (8) may be rewritten, displaying the electric field dependence explicitly, as

$$
f(\bar{k})-f^{0}\left(\epsilon_{k}\right)=-e \bar{E} \cdot \bar{\Lambda}(\bar{k}) \frac{\partial f^{0}}{\partial \epsilon_{k}}
$$


Thus, the scalar function $\mathrm{g}(\overline{\mathrm{k}})$ is replaced by a vector function $\bar{\Lambda}(\overline{\mathrm{k}})$, which has the properties of a vector mean free path. It will be shown that the BE has an exact, closedform solution for $\bar{\Lambda}$ that is valid for any strength of the applied magnetic field. This formalism provides a natural framework within which the transport properties of a real metal may be calculated.

The fundamental assumption in the work described herein is that the independentparticle Boltzmann-equation description is valid for transport processes involving electrons in metals. Three basic difficulties with this assumption are the many-body aspects of the electron-electron interaction, the inherently nonequilibrium (in the thermodynamic sense) nature of the system, and the quantization of the electron system into Landau levels by the applied magnetic field. These difficulties may not be resolved rigorously from the present state of solid-state theory, but we may present points to indicate that this approach is at least a reasonable one to take.

The questions raised by the many-body aspects of the electron-electron interactions in metals apply, at least in large part, to the existence of a Fermi surface in such systems. The long-range correlations of the electrons could be considered too strong to allow such a sharp discontinuity to exist. It appears in reality that such is not the case. Work by Migdal (ref. 28) (and discussed by Abrikosov, Gorkov, and Dzyaloshinski (ref. 29)) shows that there is a discontinuity in the electron momentum distribution in an electron gas, even in the presence of interactions. Furthermore, the existence of such sharp experimental evidence concerning the size and shape of the Fermi surfaces in a large variety of metals must be considered good evidence that this construction has some validity.

Whether the Boltzmann equation provides a valid description of transport processes in metals is a question that has received a great deal of attention. The distribution function for an electron in an independent-particle model is an approximation to a density matrix and a quantum-mechanical master-equation description of a very complex, time-varying system. Much effort has gone into attempts to understand the role of the Boltzmann equation in the framework of more general formulations of nonequilibrium thermodynamics (e.g., the work of Prigogine (ref. 30) and Edwards (ref. 31)). The role of the BE as it applies to steady-state phenomena has been extensively discussed by Dresden (ref. 1). Basically, it still remains an assumption that the Boltzmann equation is an adequate approach to steady-state processes such as electron transport in metals.

There are a large number of experiments which observe effects in metals which are quantum mechanical in origin. These include the de Haas - van Alphen oscillations in the magnetic susceptibility, de Haas - Shubnikov oscillations in magnetoresistance measurements, magnetostrictive oscillations in the physical dimensions of a metallic crystal, and many others. These effects all arise from the quantization of electron orbits in the presence of the magnetic field (i. e., the formation of the Landau levels in 
the momentum distribution). In contrast with this list of quantum effects, there is the equally valid observation that, in most cases, these quantum effects are oscillations superimposed upon usually monotonic semiclassical effects. For example, the oscillations which appear in the longitudinal magnetoresistance at high fields (the de Haas Shubnikov effect) are structure imposed on a monotonic magneiuresistance which can be calculated on the basis of the BE.

Some justification of the applicability of the semiclassical method may be obtained from a consideration of the energies involved. Three useful energies for conduction electrons in a magnetic field are the Fermi energy $\epsilon_{F}$, the width of the thermal broadening of the Fermi surface $k_{B} T$, and the separation of the quantized Landau levels $\hbar \omega=\hbar \mathrm{eH} / \mathrm{mc}$. It is convenient to take some representative numbers for comparison; for Pippard's "standard metal" (ref. 3) these energies have the following values:

$$
\begin{gathered}
\epsilon_{F}=5.57 \mathrm{eV} \\
\mathrm{k}_{\mathrm{B}} \mathrm{T}=3.62 \times 10^{-4} \mathrm{eV}(\text { at } 4.2 \mathrm{~K}) \\
\hbar \omega=1.16 \times 10^{-5} \mathrm{eV}(\text { at } 0.1 \mathrm{~T})
\end{gathered}
$$

Thus, the Fermi energy is many orders of magnitude higher than either the thermal energy or the Landau level separation, and $\epsilon_{F}$ is the dominant energy factor. Further, even at 1 tesla the level separation is only comparable to the thermal broadening. This latter comparison is further substantiated by the observed fact, mentioned previously, that even when quantum effects are present, there is still a substantial contribution which is of semiclassical origin.

In summary, then, although the use of the Boltzmann equation cannot be rigorously justified, there is every indication that it provides a valuable tool for the description of conduction processes in metals. On this basis then, we shall proceed to the formalism for the solution of the $\mathrm{BE}$ for the galvanomagnetic effects in metals.

The present work is divided into three principal segments. The following section takes the assumption of a vector mean-free-path for the Boltzmann equation, including an applied, uniform magnetic field, and traces the development of an exact, closedform solution for the conductivity tensor. As indicated previously, this solution may, in principle, be found for all forms of the Fermi surface and the scattering model; elastic scattering has been assumed for simplicity of the mathematics. The next two sections treat the implementation of the theory, the insight that a knowledge of the pertinent lattice point group may bring, and a sketch of the numerical procedures involved in performing a calculation. Finally, results are shown for two scattering models. The properties of an isotropic scattering model for a free-electron gas are 
discussed. And, then, the general implications of a degenerate kernel, as proposed by Sondheimer, are discussed with sample results for a spherical Fermi surface model.

\section{SOLUTION OF THE BOLTZMANN EQUATION}

The introduction consider ed the Boltzmann equation for conduction electrons in a metal. Steady, homogeneous electric and magnetic fields are applied to the metal, and the sample is assumed to be homogeneous with a uniform temperature distribution. In this situation, the distribution function is a function of $\bar{k}$ alone, and is written as $f(\bar{k})$, or simply $f_{k}$. When the scattering is elastic, the Boltzmann equation is

$$
\frac{e}{\hbar}\left(\bar{E}+\frac{1}{c} \vec{v}_{k} \times \vec{H}\right) \cdot \nabla_{k} f_{k}=\int Q\left(\bar{k}, \bar{k}^{\prime}\right)\left(f_{k}-f_{k^{\prime}}\right) \overline{d k^{\prime}}
$$

where the function $\mathrm{Q}(\overline{\mathrm{k}}, \overline{\mathrm{k}})$ is the (positive) transition probability per unit time for scattering from the state $\overline{\mathrm{k}}$ into the state $\overline{\mathrm{k}}^{\prime}$. For this case of elastic scattering, $\mathrm{Q}\left(\overline{\mathrm{k}}, \overline{\mathrm{k}}^{\dagger}\right)$ is symmetric (i. e., $\mathrm{Q}\left(\overline{\mathrm{k}}, \overline{\mathrm{k}}^{\prime}\right)=\mathrm{Q}(\overline{\mathrm{k}}, \overline{\mathrm{k}})$ ) and is proportional to the energyconserving $\delta\left(\epsilon_{\mathrm{k}}-\epsilon_{\mathrm{k}^{\prime}}\right)$.

The objective of the present work, as discussed in the introduction, is a solution of equation (13) that does not depend upon the precise nature of the Fermi surface or the details of the scattering mechanisms. In this section a closed-form expression for the conductivity tensor is found. (An earlier version of the formalism described in this section has appeared as ref. 32.)

The equilibrium distribution function for the electrons is the Fermi function $\mathrm{f}^{0}\left(\epsilon_{\mathrm{k}}\right)$. An applied homogeneous magnetic field does no work on the electronic system, and the effect of an electric field is to perturb the distribution function to a new function $f_{k}$. The deviation from equilibrium is assumed to be an expansion in powers of $\bar{E}$ (refs. 8 and 9), that is,

$$
f_{k}-f^{0}\left(\epsilon_{k}\right)=f^{(1)}(\bar{E})+f^{(2)}\left(E^{2}\right)+\ldots
$$

The linearization of the $\mathrm{BE}$ is obtained from the assumption that only the first term $\mathrm{f}^{(1)}(\mathrm{E})$ of equation (14) is important. This assumption results in a transport current which is also proportional to $\overline{\mathbf{E}}$, the condition of Ohm's law. The explicit form of the linearization is (refs. 8 and 9 ) 


$$
f_{k}-f^{0}\left(\epsilon_{k}\right)=-e \bar{E} \cdot \bar{\Lambda}(\bar{k}) \frac{\partial f^{0}\left(\epsilon_{k}\right)}{\partial \epsilon_{k}}
$$

The linearized Boltzmann equation for the function $\bar{\Lambda}(\overline{\mathrm{k}})$ is

$$
\left.\overline{\mathrm{v}}_{\mathrm{k}}-\frac{\mathrm{e}}{\bar{h} \mathrm{c}} \overline{\mathrm{v}}_{\mathrm{k}} \times \overline{\mathrm{H}} \cdot \nabla_{\mathrm{k}}\right) \bar{\Lambda}(\overline{\mathrm{k}})=\int \mathrm{Q}\left(\overline{\mathrm{k}}, \overline{\mathrm{k}}^{\prime}\right)\left[\bar{\Lambda}(\overline{\mathrm{k}})-\bar{\Lambda}\left(\overline{\mathrm{k}}^{\prime}\right)\right] \overline{\mathrm{dk}}^{\prime}
$$

The basic quantity describing the scattering is the positive, definite, symmetric kernel $\mathrm{Q}\left(\overline{\mathrm{k}}, \overline{\mathrm{k}}^{\prime}\right)$. It will be convenient for the later discussion to define an integral operator $Q$ such that

$$
\mathrm{Qx}(\overline{\mathrm{k}}) \equiv \int \mathrm{Q}\left(\overline{\mathrm{k}}, \overline{\mathrm{k}^{\prime}}\right) \mathrm{x}\left(\overline{\mathrm{k}^{\prime}}\right) \overline{\mathrm{dk}}{ }^{\prime}
$$

and a scalar function

$$
\mathrm{q}(\overline{\mathrm{k}}) \equiv \int \mathrm{Q}\left(\overline{\mathrm{k}}, \overline{\mathrm{k}}^{\dagger}\right) \overline{\mathrm{dk}},
$$

With the introduction of these quantities the Boltzmann equation may be written as

$$
\left.\left[(q-Q)+\frac{e}{\hbar c} \bar{v}_{k} \times \bar{H} \cdot \nabla_{k}\right)\right] \bar{\Lambda}(\bar{k})=\bar{v}_{k}
$$

The essence of the method of Taylor (refs. 8 and 9) is the use of the operator $q-Q$ to treat the scattering. No assumptions about the topology of the Fermi surface or the details of the scattering processes are needed for the solution of the BE. It has been shown by Taylor (refs. 8 and 9) that the operator $q-Q$ possesses an inverse, as long as the scattering kernel is not a $\delta$-function of $\overline{\mathrm{k}}$ and $\overline{\mathrm{k}}$, and if the constant function is excluded from the range of the operator. Both conditions can be shown to be satisfied for all problems of interest. We define the differential-integral operator

$$
\mathrm{T} \equiv \frac{\mathrm{im}}{\hbar}(\mathrm{q}-\mathrm{Q})^{-1}\left(\overline{\mathrm{v}}_{\mathrm{k}} \cdot \hat{\mathrm{h}} \times \nabla_{\mathrm{k}}\right)
$$

where $\hat{h}$ is the unit vector in the direction of the magnetic field (i. e., $\bar{H}=H \hat{h}$ ). Then, operating on the left side of equation $(19)$ by $(q-Q)^{-1}$ and defining the free-electron cyclotron frequency 


$$
\omega \equiv \frac{\mathrm{eH}}{\mathrm{mc}}
$$

result in

$$
(1-i \omega T) \bar{\Lambda}=(q-Q)^{-1} \bar{v}_{k}
$$

Equation (21) represents the Boltzmann equation for the function $\bar{\Lambda}(\bar{k})$. If a solution can be found for $\bar{\Lambda}$, then the transport properties of the system may be found from equation (15) for the distribution function. For a system with no applied magnetic field ( $\omega=0$ in eq. (21)) it was shown by Taylor (refs. 8 and 9) that there exists a solution for $\bar{\Lambda}$ in terms of eigenfunctions of the operator $q^{-1} Q$. For a nonzero magnetic field, equation (21) can be solved by forming the operator which is the inverse to 1 - iwT and operating on the left side. The inverse operator $(1-i \omega T)^{-1}$ exists since $T$, although not Hermitian, possesses a real eigenvalue spectrum (see appendix A). Hence, $1-i \omega T$ cannot be singular (ref. 33) and the inverse exists. The formal solution for $\bar{\Lambda}$ then is

$$
\bar{\Lambda}(\bar{k})=(1-i \omega T)^{-1}(q-Q)^{-1} \bar{v}_{k}
$$

The transport properties of the system, and in particular the conductivity tensor, may be determined by substitution of equation (22) into the distribution function (eq. (15)). The conductivity tensor, the quantity that describes the galvanomagnetic properties of the metal, is

$$
\sigma=-\mathrm{e}^{2} \int \overrightarrow{\mathrm{v}}_{\mathrm{k}}(1-\mathrm{i} \omega \mathrm{T})^{-1}(\mathrm{q}-\mathrm{Q})^{-1} \overline{\mathrm{v}}_{\mathrm{k}} \frac{\partial \mathrm{f}^{0}}{\partial \epsilon_{\mathrm{k}}} \overline{\mathrm{dk}}
$$

Equation (22) represents, symbolically, an exact, closed-form solution to the linearized Boltzmann equation. Thus, the conductivity tensor (eq. (23)) is an exact result, to within the linearization approximation, and is valid for any strength of the magnetic field. This generality should be contrasted with previous theories which were formulated to describe either low-field or high-field behavior. No assumption about the nature of the Fermi surface has been required, and the restriction to elastic scattering may be removed by the use of an inelastic scattering operator $P$ (refs. 8 and 9 ) rather than the $\mathrm{Q}$ of equation (17).

It is apparent that equations (22) and (23) are not in a form suitable for computation. To get a useful form for the conductivity, the quantity of interest here, we must con- 
sider the meaning of the two inverse operators in equation (22). It is shown in appendix $\mathrm{A}$ that the operator $\mathrm{T}$, although not Hermitian, does possess a spectrum of real eigenvalues and a corresponding complete set of (in general, complex) eigenfunctions. These eigenfunctions are referred to as the "magnetic scattering eigenfunctions" and form a complete set for functions in $\mathrm{k}$-space. These eigenvalues and eigenfunctions are defined by the equation

$$
\mathrm{Tb}_{l}(\overline{\mathrm{k}})=\tau_{l} \mathrm{~b}_{l} \overline{(\mathrm{k})}
$$

or, explicitly,

$$
\frac{i m}{\hbar}\left(\bar{v}_{\mathrm{k}} \cdot \hat{\mathrm{h}} \times \nabla_{\mathrm{k}}\right) \mathrm{b}_{l}(\overline{\mathrm{k}})=\tau_{l}(\mathrm{q}-\mathrm{Q}) \mathrm{b}_{\imath}(\overline{\mathrm{k}})
$$

It follows from equation (24) that $T \mathrm{bb}_{l}^{*}=-\tau_{l} \mathrm{~b}_{l}^{*}$ so that the $\mathrm{b}_{l}^{*}$ are the eigenfunctions of $\mathrm{T}$ corresponding to negative $\tau_{l}$.

In both equations (22) and (23), the operator $\mathrm{T}$ acts upon the zero-field mean free path (refs. 8 and 9$)(q-Q)^{-1} \bar{v}_{k}$. Since the $\left\{b_{l}\right\}$ form a complete set, the following expansion can be written:

$$
(\mathrm{q}-\mathrm{Q})^{-1} \mathrm{v}_{\mu}=\sum_{l} \beta_{\mu l} \mathrm{~b}_{l} \overline{(\overline{\mathrm{k}})}
$$

where $v_{\mu}$ is the $\mu^{\text {th }}$ Cartesian component of the velocity vector $\overline{\mathrm{v}}_{\mathrm{k}}$. The expansion coefficients $\beta_{\mu l}$, although they do not depend upon $\overline{\mathrm{k}}$, do depend upon the vector $\hat{\mathrm{h}}$, the direction of the magnetic field, from the definition of $T$. Note that, since the left side of equation (25) is a real function, for every term $\beta_{\mu l} \mathrm{~b}_{l}$ there must also appear the corresponding $\beta_{\mu l}^{*} \mathrm{~b}_{l}^{*}$, guaranteeing the reality of the sum.

With this expansion for the zero-field mean free path, the conductivity can be written as

$$
\sigma_{\nu \mu}=-\mathrm{e}^{2} \sum_{l} \frac{\beta_{\mu l}}{1-i \omega \tau_{l}} \int \mathrm{v}_{\nu} \mathrm{b}_{l} \overline{(\mathrm{k})} \frac{\partial \mathrm{f}^{0}}{\partial \epsilon_{\mathrm{k}}} \overline{\mathrm{dk}}
$$

The presence of the energy derivative of the Fermi function in the integral of equation (26) leads to some discussion of the $\overline{\mathrm{k}}$-dependence of functions in this theory. Because of the limit 


$$
\operatorname{Lim}_{T \rightarrow 0} \frac{\partial f^{0}}{\partial \epsilon_{k}}=-\delta\left(\epsilon_{k}-\epsilon_{F}\right)
$$

only states at the Fermi surface can contribute to the integral in equation (26). The importance of this energy $\delta$-function may be seen by consideration of a variable transformation (ref. 7 ) from the volume element $\overline{\mathrm{dk}}$ to an equivalent volume element

$$
\overline{\mathrm{dk}}=\frac{\mathrm{V}}{(2 \pi)^{3}} \mathrm{~d}^{3} \mathrm{k}=\frac{\mathrm{V}}{(2 \pi)^{3}} \frac{\mathrm{d} \epsilon_{\mathrm{k}} \mathrm{dS}}{\hbar\left|\mathrm{v}_{\mathrm{k}}\right|}
$$

where dS is the element of area on the energy shell corresponding to $\epsilon_{\mathrm{k}}$. This separation of variables into energy and surface variables, combined with the $\delta\left(\epsilon_{\mathrm{k}}-\epsilon_{\mathrm{F}}\right)$ of equation (26), implies that only functions evaluated on the Fermi surface need be considered. To simplify the notation in integrals, it is convenient to redefine the volume as

$$
\overline{\mathrm{dk}} \equiv \mathrm{d} \epsilon_{\mathrm{k}} \mathrm{d} \Omega_{\mathrm{k}}
$$

where $d \Omega_{k}$ stands for the surface integral and the multiplying factors in equation (27). After the integrations have been performed over the energy $\delta$-function, all that remains is the integral over $d \Omega_{k}$, or simply $d \Omega$, which represents an integral over the Fermi surface.

It is important to make the connection between the preceding discussion and the restriction to elastic scattering. The transition probability $Q\left(\bar{k}, \bar{k}^{\prime}\right)$ is defined for every $\overline{\mathrm{k}}$ and $\overline{\mathrm{k}}^{\prime}$ in wave-vector space. The assumption of elastic scattering restricts attention, for a given $\overrightarrow{\mathrm{k}}$, to only those $\overline{\mathrm{k}}^{\prime}$ that lie on the same energy shell. Since every $\overline{\mathrm{k}}$ is considered, the factoring of $\mathrm{k}$-space into a nested set of constant-energy surfaces is a natural view. There is no further restriction implied by the condition of elastic scattering. The functions of interest, such as $\mathrm{Q}\left(\overline{\mathrm{k}}, \overline{\mathrm{k}}^{\prime}\right), \mathrm{q}(\overline{\mathrm{k}})$, and $\bar{\Lambda}(\overline{\mathrm{k}})$, are defined off the Fermi energy surface. The discussion of the preceding paragraph is a low-temperature approximation; that is, if these functions are slowly varying functions of energy near the Fermi energy, then all k-vectors may be restricted to the Fermi surface.

After equation (26) the problem becomes the calculation of the field-orientationdependent eigenfunctions $b_{l}(\bar{k})$ and the expansion coefficients $\beta_{\mu l}$. It is apparent from equation (24) that the solution for the $\left\{b_{l}\right\}$ involves an integrodifferential equation. Fortunately, the prospect is not as dismal as it first appears, for in equation (24) the integral operator $Q$ acts upon the function $b_{l^{*}}$. Thus, if the $\left\{b_{l}\right\}$ themselves could be expanded in terms of the eigenfunctions of $\mathrm{q}^{-1} \mathrm{Q}$, the problem could be simplified. This 
simplification turns out to be quite substantial and illuminating.

Taylor, in his treatment of the magnetic-field-free Boltzmann equation (refs. 8 and 9), showed that eigenfunctions of the non-Hermitian operator $q^{-1} Q$,

$$
\left(\mathrm{q}^{-1} \mathrm{Q}\right) \mathrm{a}_{\mathrm{n}} \overline{(\overline{\mathrm{k}})}=\alpha_{\mathrm{n}} \mathrm{a}_{\mathrm{n}} \overline{(\mathrm{k})}
$$

could be defined. Appendix A includes a discussion of the properties of these scattering eigenfunctions, with their existence and completeness properties. Among their useful properties is the fact that they may be chosen to be orthonormal, so that

$$
\int \mathrm{a}_{\mathrm{r}}(\overline{\mathrm{k}}) \mathrm{q}(\overline{\mathrm{k}}) \mathrm{a}_{\mathrm{n}}(\overline{\mathrm{k}}) \mathrm{d} \Omega=\delta_{\mathrm{rn}}
$$

These scattering eigenfunctions are real, and depend only upon the nature of the Fermi surface and the electronic scattering processes, independent of the magnetic field vector.

The magnetic scattering eigenfunctions may be expanded in the $a_{n}(\bar{k})$ as follows:

$$
\mathrm{b}_{2}(\overline{\mathrm{k}})=\sum_{\mathrm{n}} \gamma_{\mathrm{ln}}(\hat{\mathrm{h}}) \mathrm{a}_{\mathrm{n}}(\overline{\mathrm{k}})
$$

where the complex expansion coefficients $\gamma_{l n}$ depend implicitly upon the field direction $\hat{\mathrm{h}}$. With the aid of this expansion and the orthogonality condition of equation (29), the eigenvalue equation for the $\tau_{l}$ becomes

$$
-i m \hat{h} \cdot \sum_{n} \gamma_{l n} \int a_{r}(\bar{k})\left[v_{k} \times \nabla_{k} a_{n} \overline{(k)}\right] d \Omega=\hbar \tau_{l}\left(1-\alpha_{r}\right) \gamma_{l r}
$$

Although this equation appears formidable, its true nature can be seen by a definition of a new set of coefficients

$$
\Gamma_{\ln } \equiv \gamma_{\ln } \sqrt{1-\alpha_{\mathrm{n}}}
$$

and the vector quantity

$$
\overline{\mathrm{C}}_{\mathrm{rn}} \equiv-\frac{\mathrm{im}}{\mathrm{h}}\left[\left(1-\alpha_{\mathrm{n}}\right)\left(1-\alpha_{\mathrm{r}}\right)\right]^{-1 / 2} \int \mathrm{a}_{\mathrm{r}}(\overline{\mathrm{k}})\left[\overline{\mathrm{v}}_{\mathrm{k}} \times \nabla_{\mathrm{k}} \mathrm{a}_{\mathrm{n}}(\overline{\mathrm{k}})\right] \mathrm{d} \Omega
$$


Thus, equation (31) has the form

$$
\hat{\mathrm{h}} \cdot \sum_{\mathrm{n}} \overline{\mathrm{C}}_{\mathrm{rn}} \Gamma_{l \mathrm{n}}=\tau_{l} \Gamma_{l \mathrm{r}}
$$

This equation represents a set of simultaneous equations, in effect a secular equation, for the $\tau_{l}$. The important feature of this equation is the fact that the only dependence upon the magnetic field is through the presence of $\vec{h}$. The matrix $\vec{C}$ is determined completely from the field-independent scattering eigenfunctions $a_{n}(\bar{k})$.

It can be seen by an integration by parts of equation (33) that the $\bar{C}$ matrix is Hermitian and, in fact, purely imaginary. This implies that the eigenvalues $\tau_{l}$ must be real. Also, since the trace of $\overline{\mathbf{C}}$ must vanish for any field direction, the $\tau_{l}$ must occur as positive-negative pairs; that is, the sum of the $\tau_{l}$ must also vanish.

The combination of equations (26) and (30) gives as an intermediate form for the conductivity

$$
\sigma_{\nu \mu}=\mathrm{e}^{2} \sum_{l, \mathrm{n}} \frac{{ }_{\mu l} \gamma_{l n} \mathrm{n}^{\mathrm{g}} \mathrm{n}_{\mathrm{n}}}{1-\mathrm{i} \omega \tau_{l}}
$$

where

$$
\mathrm{g}_{\nu \mathrm{n}}=-\int \mathrm{v}_{\nu} \mathrm{a}_{\mathrm{n}}(\overline{\mathrm{k}}) \frac{\partial \mathrm{f}^{0}}{\partial \epsilon_{\mathrm{k}}} \mathrm{d \overline { \textrm {k } }}=\int \mathrm{v}_{\nu} \mathrm{a}_{\mathrm{n}} \overline{(\overline{\mathrm{k}}) \mathrm{d} \Omega}
$$

The final reduction of the conductivity is motivated by the realization that equation (25), the expansion of $(q-Q)^{-1} v_{\nu}$ in terms of the $b_{\imath}$, is equivalent to an expansion of the same quantity in terms of the scattering eigenfunctions $a_{n}(\vec{k})$. This, in fact, was the procedure used originally (refs. 8 and 9) in the solution of the BE for the zeromagnetic-field conductivity. This double expansion results in

$$
(\mathrm{q}-\mathrm{Q})^{-1} \mathrm{v}_{\mu}=\sum_{l} \beta_{\mu l} \mathrm{~b}_{l}(\overline{\mathrm{k}})=\sum_{l} \sum_{\mathrm{n}} \beta_{\mu l} \gamma_{l \mathrm{n}^{\mathrm{a}}} \mathrm{a}^{(\overline{\mathrm{k}})}
$$

The $a_{n}$ are eigenfunctions of $q^{-1} Q$, so operating on both sides of equation (37) with $q-Q$ and using the orthogonality of the $a_{n}$ result in 


$$
\mathrm{g}_{\mu \mathrm{r}}=\sum_{l} \beta_{\mu \imath^{\gamma} \mathrm{r}_{\mathrm{r}}}\left(1-\alpha_{\mathrm{r}}\right)
$$

with the $g_{\mu r}$ defined as in equation (36). Since $\bar{C}_{r n}$ is Hermitian, the $\Gamma_{l r}$ form an orthonormal set of vectors. The replacement of $\gamma_{l r}\left(1-\alpha_{r}\right)^{1 / 2}$ by $\Gamma_{l r}$, as in equation (32), and the use of the orthogonality condition result in an expression for $\beta_{\mu l}$

$$
\beta_{\mu l}=\sum_{\mathbf{r}} \frac{\mathrm{g}_{\mu \mathrm{r}} \Gamma_{l \mathbf{r}}^{*}}{\left(1-\alpha_{\mathrm{r}}\right)^{1 / 2}}=\sum_{\mathbf{r}} \mathrm{g}_{\mu \mathrm{r}} \gamma_{l \mathbf{r}}^{*}
$$

This result allows the final simplification of the expression for the conductivity. From equation (35), then,

$$
\sigma_{\nu \mu}=\mathrm{e}^{2} \sum_{l} \frac{1}{1-\mathrm{i} \omega \tau_{l}} \sum_{\mathrm{r}, \mathrm{n}} \frac{\mathrm{g}_{\mu \mathrm{r}} \Gamma_{l \mathrm{r}}^{*} \Gamma_{l \mathrm{n}} \mathrm{g}_{\nu \mathrm{n}}}{\left(1-\alpha_{\mathrm{r}}\right)^{1 / 2}\left(1-\alpha_{\mathrm{n}}\right)^{1 / 2}}
$$

or

$$
\sigma_{\nu \mu}=\mathrm{e}^{2} \sum_{l} \frac{1}{1-\mathrm{i} \omega \tau_{l}} \sum_{\mathrm{r}, \mathrm{n}} \mathrm{g}_{\mu \mathrm{r}} \gamma_{l \mathrm{r}}^{*} \gamma_{l \mathrm{n}} \mathrm{g}_{\nu \mathrm{n}}
$$

It is not immediately apparent that either form for the conductivity is closely akin to the usual results (ref. 3) presented on the basis of phenomenological arguments. Any detailed comparison would involve some specification of a model for the Fermi surface and would not have the general validity of the preceding discussion. The desired comparison may be helped by the recognition that the magnetic scattering eigenvalues $\tau_{l}$ occur in positive-negative pairs. In fact, as may be verified from equations (33) and (34), if $\Gamma_{l \mathrm{n}}$ is an eigenvector corresponding to an eigenvalue $\tau_{l}$, then $\Gamma_{l \mathrm{n}}^{*}$ is also an eigenvector with the associated eigenvalue $-\tau_{l}$. Thus, if the sum over $l$ in equation (39) is restricted to only include the positive values of $\tau_{l}$, the final result is 


$$
\begin{aligned}
\sigma_{\nu \mu}=\mathrm{e}^{2} \sum_{l} \sum_{\mathbf{r}, \mathbf{n}} 2 \mathrm{~g}_{\mu \mathrm{r}} \mathrm{g}_{\nu \mathrm{n}} \operatorname{Re}\left(\gamma_{l \mathrm{r}}^{*} \gamma_{l \mathrm{n}}\right) \quad \text { for } \tau_{l}=0 \\
+\mathrm{e}^{2} \sum_{l} \sum_{\mathrm{r}, \mathrm{n}} \frac{2 \mathrm{~g}_{\mu \mathrm{r}} \mathrm{g}_{\nu \mathrm{r}}}{1+\left(\omega \tau_{l}\right)^{2}}\left[\operatorname{Re}\left(\gamma_{l \mathrm{r}}^{*} \gamma_{l \mathrm{n}}\right)-\omega \tau_{l} \operatorname{Im}\left(\gamma_{l \mathrm{r}}^{*} \gamma_{l \mathrm{n}}\right)\right] \text { for } \tau_{l} \neq 0
\end{aligned}
$$

\section{SYMMETRY PROPERTIES OF THE SCATTERING EIGENFUNCTIONS}

It was shown in the preceding section that a formal, closed-form solution to the linearized Boltzmann equation exists for all magnetic field strengths and orientations. It was also demonstrated that this solution could be obtained by using two eigenfunction expansions. For any application of these results to a real metal these eigenvalue problems must be formulated in terms of the electronic scattering processes and band structure. This places a limit upon the number of general observations which may be drawn from the formalism. Fortunately, a large amount of information about the scattering eigenfunctions (referring now to both eigenvalue problems) and the methods of computation may be obtained from the symmetry properties of the system. In this section the application of group theory to determining the properties of the scattering eigenfunctions is discussed.

The difference between a free-electron gas and the system of conduction electrons in an independent-particle model for a real metal is the anisotropy forced upon the conduction electrons by the lattice of the ion cores in the metal. For many metals the most striking example of this anisotropy is the shape of the Fermi surface, the constantenergy surface which, at absolute zero, defines the boundary between filled and unfilled electronic states in $\mathrm{k}$-space. For some metals, such as aluminum, the Fermi surface is very complex and is composed of several separate sheets; for copper, the Fermi surface is a single sheet but is distorted from the free-electron sphere sufficiently to touch the boundary of the Brillouin zone. This contact provides a path through $\mathrm{k}$-space for electrons, giving rise to the concept of an "open" orbit for conduction. The common ground between these different cases is the influence the symmetry of the lattice has upon the electrons. The lattice has the symmetry properties of a specified point group, and the Fermi surface also has the properties of the same point group, with respect to rotations and rotatory inversions in reciprocal space. The properties of the conduction electrons must also be governed by this point group, and the scattering eigenfunctions may be considered from this viewpoint.

Consider a Fermi surface whose symmetry properties are those of a point group $\mathscr{G}$. This group consists of some number $\mathrm{g}$ of rotations and possibly rotatory inversions, 
each operation symbolized by the operator $\mathscr{R}$. This group has a number of irreducible representations which give a set of finite matrices to represent the operations. If the $\alpha^{\text {th }}$ irreducible representation of $\mathscr{G}$ has the dimension of $\mathrm{n}_{\alpha}$, then the matrices for this irreducible representation are square and have dimension $\mathbf{n}_{\alpha}$, and the matrix elements are represented by the symbol $\mathrm{D}_{\mathrm{ij}}^{(\alpha)}(\mathscr{R})$. The effect of a group operation acting upon a function of $\overline{\mathbf{k}}$ is defined by

$$
\mathscr{R} \mathrm{f}(\overline{\mathrm{k}})=\mathrm{f}\left(\mathrm{R}^{-1} \overline{\mathrm{k}}\right)
$$

where $\mathscr{R}$ is symbolic for the abstract group operator and $\mathrm{R}$ is the $3 \times 3$ matrix representation of $\mathscr{t}$ which acts upon the triplet of numbers $\left(\mathrm{k}_{\mathrm{x}}, \mathrm{k}_{\mathrm{y}}, \mathrm{k}_{\mathrm{z}}\right)$.

Since the scattering in the linearized approximation is independent of the magnetic field, the $\mathrm{Q}\left(\overline{\mathrm{k}}, \overline{\mathrm{k}}^{\dagger}\right)$ must reflect the symmetry of the lattice point group. The only assumption about the scattering kernel which must be made is a weak restriction, particularly in view of the restrictions other authors (ref. 24) have made. Consider the scattering from a state $\overline{\mathrm{k}}$ to (or from) a state $\overline{\mathrm{k}}^{\prime}$. If there are no preferred directions in space imposed by some external source, then this scattering process should be identical to the scattering from $R \bar{k}$ to $R \bar{k}$ ', where $\bar{k}$ and $R \bar{k}$ are equivalent points. The sense of equivalence here is simply that the point $R \bar{k}$ is obtained from the point $\bar{k}$ through one of the group operations $\mathscr{x}$. Thus, for the group of order $g$, a general point on the Fermi surface will have $g$ distinct images under the group operations, including the identity operation. This equivalence assumption about the scattering kernel may be formally written as

$$
\mathrm{Q}\left(\overline{\mathrm{k}}, \overline{\mathrm{k}}^{\prime}\right)=\mathrm{Q}\left(\mathrm{R} \overline{\mathrm{k}}, \mathrm{R} \overline{\mathrm{k}}^{\prime}\right)
$$

for all $\overline{\mathrm{k}}$ and $\overline{\mathrm{k}}$, and all $\mathscr{R} \in \mathscr{G}$. This assumption does not require that $\mathrm{Q}\left(\overline{\mathrm{k}}, \overline{\mathrm{k}} \mathrm{k}^{\mathrm{r}}\right)$ be degenerate or that it only depend upon $\overline{\mathrm{k}}-\overline{\mathrm{k}}^{\prime}$ and, in fact, is a very weak restriction upon the form of the scattering kernel. The fact is, the use of this assumption and the powerful tools of group representation theory allow interpretation and understanding of the scattering eigenvalue problem (eq. (28)).

For the purposes of the problems considered here, the most important result from group representation theory is the concept of a basis function of an irreducible representation. The formal definition of a basis function is as follows: If the $\alpha^{\text {th }}$ irreducible representation of a group $\mathscr{G}$ has the dimension $\mathrm{n}_{\alpha}$, then there exist $\mathrm{n}_{\alpha}$ basis functions $\psi_{i}^{(\alpha)}$ such that, for any $\mathscr{P}$ in $\mathscr{G}$,

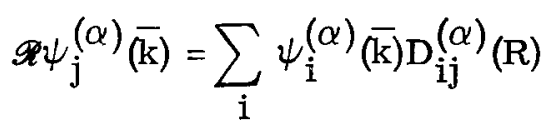


To make the connection between the scattering eigenvalue problem and the basis function the following theorem is important:

Theorem I: The integral operator $Q$ of equation (22) commutes with all $\mathscr{A}$ of $\mathscr{G}$, that is,

$$
\mathscr{R}(\mathrm{Qf})=\mathrm{Q}(\mathscr{R f})
$$

Proof: By the definition of $\mathrm{Q}$,

$$
\begin{aligned}
\mathscr{R}(\mathrm{Qf}) & =\mathscr{R} \int \mathrm{Q}\left(\overline{\mathrm{k}}, \overline{\mathrm{k}}^{\prime}\right) \mathrm{f}\left(\overline{\mathrm{k}}^{\prime}\right) \mathrm{d} \Omega^{\prime} \\
& =\int \mathrm{Q}\left(\mathrm{R}^{-1} \overline{\mathrm{k}}, \overline{\mathrm{k}}^{\prime}\right) \mathrm{f}\left(\overline{\mathrm{k}}^{\prime}\right) \mathrm{d} \Omega^{\prime} \quad \text { from equation (40) } \\
& =\int \mathrm{Q}\left(\overline{\mathrm{k}}, \mathrm{R}^{\prime}\right) \mathrm{f}\left(\overline{\mathrm{k}}^{\prime}\right) \mathrm{d} \Omega^{\prime} \quad \text { from equation (41) }
\end{aligned}
$$

but $f\left(\bar{k}^{\prime}\right)=f\left(R^{-1} \bar{R}^{\prime}\right)=\mathscr{R} f\left(\mathrm{R}^{\prime}\right)$ and the energy of $R \bar{k}^{\prime}$ is the same as the energy of $\overline{\mathrm{k}^{\prime}}$ due to the invariance of the Fermi surface. Thus,

$$
\begin{aligned}
\mathscr{R}(\mathrm{Qf}) & =\int \mathrm{Q}\left(\overrightarrow{\mathrm{k}}, \mathrm{R}^{\prime}\right) \mathscr{R} \mathrm{f}\left(\mathrm{R}_{\mathrm{k}^{\prime}}\right) \mathrm{d} \Omega^{\prime} \\
& =\mathrm{Q}(\mathscr{R} \mathrm{f})
\end{aligned}
$$

All $\overline{\mathrm{k}}$ vectors have been taken to be vectors to the Fermi surface, to be consistent with the discussion of the preceding section. All integrals are taken over the Fermi surface. This assumption is not as restrictive as it appears, since the energy integral implies an integral over all the energy shells. Each constant-energy shell has the same symmetry properties as the Fermi surface, and, thus, the generalization is immediate.

Theorem I implies that there is a connection between the scattering eigenfunctions of equation (28) and the set of basis functions of the group representations. The establishment of this connection, along with further implications, is the object of this section.

The scattering eigenvalue problem (eq. (28) or, in practice, eq. (A1)) is in reality a Fredholm integral equation, a homogeneous equation of the second kind (ref. 22). It is a result of a theorem in the theory of integral equations known as Mercer's theorem that, since $\mathrm{Q}\left(\overline{\mathrm{k}}, \overline{\mathrm{k}}^{\prime}\right)$ is a positive definite, continuous, symmetric kernel, there exists a biharmonic expansion for $Q$ in terms of its eigenfunctions (ref. 23). The translation of this theorem requires a little groundwork.

The spherical harmonic functions $\mathrm{Y}_{l}^{\mathrm{m}}(\theta, \varphi)$ form a complete orthonormal set of functions for functions defined on the unit sphere. Another property of the spherical 
harmonics, perhaps not so widely recognized, is that they are basis functions of the rotation group. The $\mathrm{Y}_{l}^{\mathrm{m}}$ obey an equation analogous to equation (42) (ref. 34) with $l$ labeling the irreducible representations and $m$ denoting the partners of the basis. Since the $l^{\text {th }}$ irreducible representation of the rotation group has dimension $2 l+1$, there are $2 l+1$ partners in a basis set, corresponding to the $2 l+1$ values of $\mathrm{m}$ for a given $l$ in a spherical harmonic.

Now consider a kernel $Q\left(\bar{k}, \bar{k}^{\prime}\right)$, where $\bar{k}$ and $\bar{k}^{\prime}$ are vectors to the unit sphere. If this kernel is restricted to being spherically symmetric (i.e., the function $Q$ may depend only upon the difference $\left|\bar{k}-\bar{k}^{\prime}\right|$ ), then there exists an expansion of $Q$ in terms of the spherical harmonics

$$
\mathrm{Q}\left(\overline{\mathrm{k}}, \overline{\mathrm{k}^{\prime}}\right)=\sum_{l=0} \mathrm{C}_{l} \sum_{\mathrm{m}} \mathrm{Y}_{l}^{\mathrm{m}} \overline{(\mathrm{k})} \mathrm{Y}_{l}^{\mathrm{m}}{ }^{*}\left(\overline{\mathrm{k}^{\prime}}\right)
$$

Note that the existence of this expansion does not imply that the kernel must be degenerate, in which case the sum would have to be finite. The implication in equation (44) is that the function $Q$ is well approximated by the sum on the right side if a sufficiently large number of $l$-values are included.

The purpose is to find an expansion, similar to equation (44), for a scattering kernel which is restricted to having the symmetry of a finite point group, as specified by equation (41). Obviously, the functions analogous to the spherical harmonics (i. e., a complete, orthonormal set of basis functions of the point group $\mathscr{G}$ ) are required.

The existence and methods of obtaining such functions, known generally as symmetry-adapted functions, are sufficiently detailed in the literature ( $r e f .35$ ) not to require an exhaustive treatment here. Given a group $\mathscr{G}$ (meaning that we have all the matrices of all the irreducible representations of $\mathscr{G}$ ), consider one of the irreducible representations, labeled by $\alpha$, which is of dimension $\mathrm{n}_{\alpha}$. Since $\mathscr{G}$ is assumed to be a point group, it is a subgroup of the full rotation group, including inversions, if necessary. The representations of the rotation group are, in general, reducible with respect to $\mathscr{G}$. This means that the $l^{\text {th }}$ representation of the rotation group may contain more than one representation of $\mathscr{G}$. If the irreducible representation is contained in the $l^{\text {th }}$ representation, then there are $\mathrm{n}_{\alpha}$ linear combinations of $\mathrm{Y}_{l}^{\mathrm{m}}$, summed over $\mathrm{m}$, that are basis functions for the $\alpha$ representation of $\mathscr{G}$. The set of all such linear combinations, for all $l$ and all $\alpha$ of $\mathscr{G}$, form a complete orthonormal set of functions.

The symmetry-adapted functions for the full cubic group $O_{h}$ have long been known and tabulated as the Kubic harmonic functions (ref. 36). In principle, the same constructions may be performed for each crystal group (in particular, see Altmann and Cracknell (ref. 37)) and all such tabulations could be kept as ready reference. However, all that is required is the existence of such a complete orthonormal set of func- 
tions $\mathrm{x}_{l}^{\mathrm{jr}}$ indexed by the angular momentum of the contributing spherical harmonics $l$, the label of the irreducible representation of $\mathscr{G} \mathrm{j}$, and the column (or the component) of the representation $r$.

Consider a kernel function $\mathrm{Q}\left(\overline{\mathrm{k}}, \overline{\mathrm{k}}^{\prime}\right)$, where $\overline{\mathrm{k}}$ and $\overline{\mathrm{k}}^{\prime}$ are vectors to the Fermi surface, which is to be invariant under the operations of a point group, as discussed earlier in this section. Then there exists an expansion for $Q$, analogous to equation (44), in terms of the symmetry-adapted functions. This expansion is the content of theorem $\Pi$, as follows:

Theorem III: Given a function $\mathrm{Q}\left(\overline{\mathrm{k}}, \overline{\mathrm{k}}^{\prime}\right)$ which is invariant under all operations $\mathscr{x}$ of a group $\mathscr{G}\left(\right.$ i. e., $\mathrm{Q}\left(\overline{\mathrm{k}}, \overline{\mathrm{k}}^{\prime}\right)=\mathrm{Q}\left(\mathrm{R} \overline{\mathrm{k}}, \mathrm{R}^{\prime}\right)$ for all $\mathscr{A}$ in $\left.\mathscr{G}\right)$, then there exists the expansion

$$
\left.Q\left(\bar{k}, \bar{k}^{\prime}\right)=\sum_{l, l^{\prime}, j} A_{l l^{\prime}}^{\mathrm{j}} \sum_{r} x_{l}^{\mathrm{jr}} \overline{(\mathrm{k})} \mathrm{X}_{l^{\prime}}^{\mathrm{j} \mathrm{r} *} \overline{(\mathrm{k}}^{\prime}\right)
$$

The proof of this theorem is straightforward, but not instructive for the purposes of this report, and so it is given in appendix $B$. The important fact to note is that equation (45) does not represent a treatment of the scattering by a degenerate kernel, since the sums of $l$ and $l^{\prime}$ are infinite. Equation (45) does however, indicate, simplifications which arise if the kernel is degenerate. Further evidence of this point appears in the section DEGENERATE KERNEL THEORY.

The groundwork is now laid to make the connection with theorem $I$. It is apparent that the eigenfunctions of the scattering operator must be related to symmetry-adapted functions. This connection is provided by the content of theorem III:

Theorem III: A function $\mathrm{y}_{\mathrm{j}}^{\mathrm{r}}(\overrightarrow{\mathrm{k}})$ is a basis function for the group $\mathscr{g}$; that is, it satisfies the equation

$$
\mathscr{x} \mathrm{y}_{\mathrm{j}}^{\mathrm{r}}(\overline{\mathrm{k}})=\sum_{\mathrm{r}^{\prime}} \mathrm{y}_{\mathrm{j}}^{\mathrm{r}^{\prime}} \overline{(\mathrm{k}) \mathrm{D}_{\mathrm{rr}}^{\mathrm{j}}} \mathrm{j}^{\mathrm{R})}
$$

if and only if

$$
\mathrm{y}_{\mathrm{j}}^{\mathrm{r}} \overline{(\mathrm{k})}=\sum_{l} \mathrm{~b}_{l} \mathrm{x}_{l}^{\mathrm{jr}} \overline{(\mathrm{k})}
$$

The proof of this theorem is also given in appendix $B$, since it is merely an exercise in group representation theory. Equation (46) is the same as equation (42), although the notation has been changed to make the following discussion smoother. 
Now that the connection between the symmetry-adapted functions and the scattering kernel has been established, the scattering eigenvalue problem may be reformulated. Since any kernel $Q$ may be expanded as in equation (45), the modified scattering kernel of equation (A1) of appendix A clearly has such an expansion. It is shown in appendix B that the function $q(\overline{\mathrm{k}})$ is a scalar with respect to the group operations, so that the modified scattering kernel has the same symmetry properties as the original kernel $Q\left(\bar{k}, \overline{\mathrm{k}}^{\prime}\right)$. Formally then,

$$
\frac{\mathrm{Q}\left(\overline{\mathrm{k}}, \overline{\mathrm{k}}^{\prime}\right)}{\sqrt{\mathrm{q}(\overline{\mathrm{k}}) \mathrm{q}\left(\overline{\left.\mathrm{k}^{\prime}\right)}\right.}}=\sum_{l, l^{\prime}, \mathrm{j}} \mathrm{A}_{l l^{\prime}}^{\mathrm{j}} \sum_{\mathrm{r}} \mathrm{x}_{l}^{\mathrm{jr}} \overline{(\mathrm{k})}\left(\mathrm{x}_{l^{\prime}}^{\mathrm{j} \mathrm{r}^{*}}\right) \overline{\left(\mathrm{k}^{\prime}\right)}
$$

The relation between the basis functions $\mathrm{y}_{\mathrm{j}}^{\mathrm{r}}$ and the symmetry-adapted functions $\mathrm{x}_{l}^{\mathrm{j} r}$ (eq. (47)) is used to derive a secular equation for the scattering eigenvalues $\alpha$, as in equation (A1),

$$
\sum_{l^{\prime}}\left(\mathrm{A}_{l l^{\prime}}^{\mathrm{j}},-\alpha \delta_{l l^{\prime}}\right) \mathrm{b}_{l^{\prime}}=0
$$

A consequence of theorem $I I$, as shown in appendix $B$ for real, symmetric kernels, is that the matrix of expansion coefficients A must be Hermitian. Thus, the scattering eigenvalues, the roots of equation (49), must be real, confirming the results of appen$\operatorname{dix} \mathrm{A}$; and the vectors of the expansion coefficients, the $b_{l}$, must either be automatically orthogonal (for distinct eigenvalues), or else be constructed to be orthogonal (for degenerate eigenvectors). Since the scattering eigenfunctions, labeled by $a_{j}^{r}$ according to the notation of equations (47) and (28), are related to the eigenfunctions of equation (48) through the scalar function $\sqrt{\mathrm{q}(\overline{\mathrm{k}})}$, the transformation properties of the two functions will be the same.

The meaning of the notation and the scattering eigenvalues can be seen by looking at the structure of the matrix A of equation (48). This coefficient matrix connects only symmetry functions which belong to the same irreducible representation of the group. In this sense, $A$ is a diagonal block matrix of block dimension $g$ and each block, individually Hermitian, is indexed by its irreducible representation. Each block will be of infinite dimension if the kernel is not degenerate and its eigenvalues are the scattering eigenvalues. Each scattering eigenvalue must carry two indices, one corresponding to the irreducible representation and one corresponding to an ordinal number within its block. There is one complication which is partially hidden in this indexing. If the $\alpha$ irreducible representation of $\mathscr{G}$ has the dimension $\mathrm{n}_{\alpha}$, then each eigenvalue corre- 
sponding to the block $\alpha$ must be $\mathrm{n}_{\alpha}$-fold degenerate, corresponding to the $\mathrm{n}_{\alpha}$ partners of the set of basis functions.

The possibility of providing an indexing and a characterization of the symmetry properties of the scattering eigenfunctions allows an insight into the meaning and structure of the formal equations derived in the preceding section. The fact that it also provides an aid in the numerical treatment of the problem will be consider ed in the next section. For the time being, consider two major steps in the formalism, the formation of the $\overline{\mathrm{C}}$-matrix, (eq. (33)) and the definition of the $\mathrm{g}_{\nu \mathrm{n}}$ factors of equation (36). Use of the tools of group representation theory allows some strong statements to be made concerning these equations.

First, consider the definition of the $\mathrm{g}_{\nu \mathrm{n}}$, but with the indexing of the scattering eigenfunctions indicated explicitly

$$
\mathrm{g}_{\nu l}=-\int \mathrm{v}_{\nu}(\overline{\mathrm{k}}) \mathrm{a}_{\mathrm{j}}^{\mathrm{r}}, \mathrm{n} \overline{(\overline{\mathrm{k}}) \mathrm{d} \Omega}
$$

The indices $\mathbf{r}$ and $\mathrm{n}$ on a refer to the column of the representation and the ordinal number of the eigenvalue, respectively. The index $l$ on the $g$ is meant to include schematically all possible indices from the a. For convenience, let us consider the full cubic group $\mathrm{O}_{\mathrm{h}}$ of order 48 with 10 distinct irreducible representations, having dimensions from 1 to 3 . The velocity vector $\bar{v}_{\mathrm{k}}$ will have specified transformation properties for this group; in fact, $\bar{v}_{k}$ transforms according to the $\Gamma_{15}$ irreducible representation (ref. 38). The selection rule for the integral in equation (50) may be obtained from one of two approaches. Either the integral is formally evaluated by summing over the contributions of the 48 equivalent zones of the Fermi surface, or else the content of the Kronecker product $\mathscr{D}(\overline{\mathrm{v}}) \otimes \mathscr{D}\left(\mathrm{a}_{\mathrm{j}}\right)$ is examined for a scalar part. The script $\mathscr{D}$ is used to symbolize the irreducible representation of the indicated quantities. The former approach is used in the following section, but the latter is more useful for the investigation of the selection rules.

The appropriate guide to use is the result (ref. 39) that an integral of the type shown in equation (50) will vanish unless there is some part of the integrand which is a scalar under the group operations. Since, for $O_{h}, \bar{v}_{k}$ is known to transform as $\Gamma_{15}$, all that is necessary is to scan a table of Kronecker products of $\Gamma_{15}$ with all irreducible representations of $O_{h}$ to discover the fact that only $\Gamma_{15} \otimes \Gamma_{15}$ contains the identity $\Gamma_{1}$. Even further, the integral will vanish for a scattering eigenfunction which is $\Gamma_{15}$ unless the column index of the function is the same as the column index of the velocity (i.e., the component of $\left.\bar{v}_{k}\right)$. Thus,

$$
\mathrm{g}_{\nu l}=-\int \mathrm{v}_{\nu}(\overline{\mathrm{k}}) \mathrm{a}_{\mathrm{j}}^{\mathrm{r}, \mathrm{n}} \overline{(\mathrm{k})} \mathrm{d} \Omega \delta_{\mathrm{j}, \mathscr{D}(\overline{\mathrm{v}})}{ }^{\delta} \mathrm{r} \nu
$$


By the same token, the selection rules for the formation of the $\bar{C}$-matrix (eq. (36)) can by obtained by looking at a triple Kronecker product of representations. This should not be striking since $\bar{C}$ is really the matrix of the matrix elements of a crystal angular momentum operator $\bar{v}_{\mathbf{k}} \times \nabla$ taken with respect to the scattering eigenfunctions. This is a familiar problem in nuclear physics, and its solution is the set of Racah coefficients for the scalar content of the triple product.

In the full cubic group the angular momentum transforms as the $\Gamma_{15}^{\prime}$ irreducible representation and thus has even parity under inversions. A table of the triple product of $\Gamma_{15}^{\prime}$ with all the irreducible representations will show which products contain the identity representation. Conversely, for the products which do not contain the identity, the matrix element of $\bar{C}$ must vanish. Even without such a product table, the angular momentum cannot have matrix elements between states of different parity, since the product must then have odd parity under inversion. Thus, the only contribution to the gross transport properties arises from the part of the scattering kernel which has odd parity, because of the nature of the $\mathrm{g}_{\nu l}$. The qualification implied here is necessary, since the effective anisotropic relaxation time $\mathrm{q}^{-1}(\overline{\mathrm{k}})$ is determined from the scalar part of $Q\left(\bar{k}, \bar{k}^{\prime}\right)$ and determines the scale for the system.

The importance of the symmetry properties in determining the transport properties of electrons in a metal should be apparent at this point. The representation theory of finite groups provides powerful tools for the understanding of the formalism discussed in the preceding section. The validity of an even stronger statement, that the use of group theory is essential for the numerical description of the galvanomagnetic properties of a real metal, is discussed in the next section.

\section{STRUCTURE OF THE CALCULATIONS}

The desired product of this investigation is a computational procedure capable of giving the galvanomagnetic properties of any metal. Equations (38) or (39) describe the conductivity of a metal, on the basis of an exact solution of the Boltzmann equation. The implementation of this formalism to a real metal involves a number of computational problems, which are considered in this section.

There are two primary areas for comparison with experimental results, the Fermi surface construction and the scattering model. When a galvanomagnetic experiment is used as a topological probe of a Fermi surface, such as that of copper (ref. 40), the details of the scattering model are not as important as an accurate construction of the Fermi surface and velocity vectors. On the other hand, as mentioned in the introduction, galvanomagnetic experiments have been supplanted, for the most part, by other types of experiments for topological investigations. The ability to investigate the effect 
of different scattering models upon the galvanomagnetic properties of metals becomes a more important role. The input information to a calculation consists a construction of the Fermi surface $\epsilon_{F}(\bar{k})$, the Fermi velocity $\bar{v}_{F}(\bar{k})$, and the scattering model $\mathrm{Q}\left(\overline{\mathrm{k}}, \overline{\mathrm{k}}^{\prime}\right)$.

The calculation in which equation (38) is evaluated may be divided into two distinct parts. It should be apparent from the discussion of the section SOLUTION OF THE BOLTZMAN EQUATION that the magnetic field plays no essential role in the theory until the determination of the magnetic scattering eigenvalues and eigenfunctions $\tau_{l}$ and $b_{l}$ is desired. The computations then may be classified as field-free or field-dependent. In the remainder of this section, the numerical procedures used to implement the theory are described in sequence.

The computation that requires the most time and accuracy is the solution of equation (38) for the scattering eigenvalues and eigenfunctions. Even when the scattering is limited to Fermi surface states, as assumed herein, the numerical problem is imposing. Equation (38), or, more properly, equation (A1), is a homogeneous Fredholm integral equation. Standard iterative procedures exist for the solution of such equations (ref. 22). The recognition that standard techniques are available for a solution does not reduce the magnitude of the numerical problem. To cover the Fermi surface with a mesh having a $5^{\circ}$ separation between points would require approximately 2500 points. A complete knowledge of the scattering kernel $Q(\bar{k}, \bar{k})$, with no simplifications from the symmetry, would then require $(2500)^{2}$ values to be stored. It is obvious that this is a number larger than the fast-access storage of any computer now in use. Fortunately, the properties of the covering point group allow drastic simplifications in the problem.

Let us consider, where possible, the specific example of the full cubic group $\mathrm{O}_{\mathrm{h}}$ of ordre 48. Since there are 48 elements in the group, only one forty-eighth of the Fermi surface is necessary to completely determine the entire surface. By the same token, a set of basis functions for an irreducible representation of $O_{h}$ need be specified only in one forty-eighth of the surface in order to compute the functions over the entire surface. It was shown previously that the eigenfunctions of equation (A1) transform as basis functions of the irreducible representations of the reciprocal lattice point group. The degeneracy of an eigenfunction is the same as the dimension of the pertinent irreducible representation. This prior knowledge of the symmetry properties of the scattering eigenfunctions allows the scattering eigenvalue problem to be reduced to a problem over one forty-eighth of the Fermi surface. Consider the eigenfunction of equation (A1) $\mathrm{Y}_{\Gamma}^{i}(\overline{\mathrm{k}})$, which transforms as the $i^{\text {th }}$ column of the $\Gamma$ irreducible representation of $\mathrm{O}_{\mathrm{h}}$. The matrices of $\Gamma$ have the dimension $\mathrm{n}_{\Gamma}$ so that the eigenvalue corresponding to this eigenfunction is $\mathrm{n}_{\Gamma}$-fold degenerate. Rather than iterating equation (1) over the entire Fermi surface to obtain each eigenfunction, it is only necessary to note that $\mathrm{Y}_{\Gamma}^{\mathrm{i}}$ is the solution to the reduced scattering problem 


$$
\left.\alpha \mathrm{Y}_{\Gamma}^{\mathrm{i}} \overline{(\overline{\mathrm{k}})}=\sum_{\mathrm{j}=1}^{\mathrm{n}_{\Gamma}} \int_{\mathrm{IZ}} \mathrm{Y}_{\Gamma^{\mathrm{i}}}^{\mathrm{i}} \overline{\left.\mathrm{k}^{\prime}\right)} \mathscr{Q}_{\mathrm{j} \mathrm{i}}^{\Gamma} \overline{(\mathrm{k},}, \overline{\mathrm{k}^{\prime}}\right) \mathrm{d} \Omega^{\prime}
$$

The integral in equation (52) is over all $\overline{\mathbf{k}}^{\prime}$ within one forty-eighth of the Fermi surface, the irreducible zone (IZ); and the kernel $\mathscr{Q}_{\mathrm{ji}}^{\Gamma}\left(\overline{\mathrm{k}}, \overline{\mathrm{k}}^{\prime}\right)$ is an $\mathrm{n}_{\Gamma} \times \mathrm{n}_{\Gamma}$ matrix defined as

$$
\mathscr{Q}_{j i}^{\Gamma}=\sum_{\mathrm{R}} D_{\mathrm{ji}}^{(\Gamma)}(\mathrm{R}) \frac{\mathrm{Q}\left(\overline{\mathrm{k}}, \mathrm{R}^{\mathrm{k}^{\prime}}\right)}{\sqrt{\mathrm{q}(\overline{\mathrm{k}}) \mathrm{q}\left(\overline{\left.\mathrm{k}^{\prime}\right)}\right.}}
$$

Equation (52) is an integral equation that is equivalent to equation (A1), with $\overline{\mathrm{k}}$ and $\overline{\mathrm{k}}^{\prime}$ restricted to lie within the IZ. Interation of equation (52) gives the scattering eigenvalues and eigenfunctions.

The savings in time and storage locations that are allowed by this reformulation of the problem should be apparent. The convenient choice of 66 points in the IZ, equivalent to over 3000 points on the entire surface, entails the storage of $\left(\mathrm{n}_{\Gamma}\right)^{2} \times(66)^{2}$ values of $\mathscr{Q}_{i j}$ rather than (3000) ${ }^{2}$ values of $Q$. Since the time to perform one iteration is proportional to the square of the number of mesh points, this reduction to the IZ also provides a substantial savings in computer time.

The method of solution chosen is known as Kellogg's method (ref. 22). An initial choice for the set of functions $\left\{\mathrm{Y}_{\Gamma}^{\mathbf{i}}\right\}$ is iterated by means of the integral in equation (52). The eigenvalue is approximated by the ratio of the norms of successive iterates. Once satisfactory convergence to an eigenvalue has been obtained, a process which takes relatively few iterations, the content of the kernel due to this eigenvalue (ref. 22) is subtracted from the kernel. The iterative process then is restarted. One feature of this method is the use of a pseudorandom number generator to provide the initial set of functions. This choice eliminates the possibility of a trial function that is accidentally orthogonal to the eigenfunctions of a reduced kernel.

Once the scattering eigenvalues and eigenfunctions have been obtained, the next step is the calculation of the $\overline{\mathrm{C}}$ matrix (more properly, the set of three matrices which have been symbolized by the vector notation for $\overline{\mathrm{C}}$ ) defined by equation (33). As discussed in the preceding section, the selection rules for the formation of the matrix elements of $\bar{C}$ are obtainable from the content of the triple Kronecker product of the irreducible representations participating. One aspect of this observation was that equation (52) need only be solved for the eigenfunctions which have odd parity under inversion, since only these functions can give any contribution to the transport properties. 
The only numerical problem involved in the construction of the $\bar{C}$ matrices (still a field-free problem) lies in the formation of the gradients of the scattering eigenfunctions. Numerical differentiation by some type of finite-difference method is a very inaccurate process on a computer. To avoid this inaccuracy, the scattering eigenfunctions are approximated by the formation of interpolation polynomials formed from the appropriate Kubic harmonics (the symmetry-adapted functions for the cubic group). Since the interpolation polynomials may be differentiated analytically, the finite-difference method is not necessary. Thus, once the factors of the integrand of equation (33) are known, it only remains to either perform the integrals numerically for all possible products allowed by the selection rules, or else use a table of Racah coefficients for the point group to form the scalar parts of the integrands, with the number of numerical integrations being correspondingly reduced.

The preceding discussion covers the field-free portion of the calculations. The numerical procedures involved are the most time- and storage-consuming of the entire problem. The important point to remember is that these calculations need be done only once for a given Fermi surface and scattering model. Once a Fermi surface construction is made and a scattering model formulated, the scattering eigenvalue problem can be solved and the $\overline{\mathrm{C}}$ matrix and the $\mathrm{g}_{\nu \mathrm{n}}$ factors calculated, and a very large amount of information is available for use. That such a division of labor exists is clearly a result of the linearization procedure used to simplify the Boltzmann equation. In this approximation, the scattering term is independent of the electric and magnetic fields. To proceed to the next order in the Boltzmann equation would certainly introduce substantial difficulties.

The remainder of the calculation, the field-dependent part, presents no essential problem and requires no major computational effort. Once a field direction $\hat{h}$ had been chosen, the scalar product of $\hat{h}$ with the $\vec{C}$ matrix results in a purely imaginary Hermitian matrix which must be diagonalized. For convenience this resultant matrix is treated as a real, antisymmetric matrix; the imaginary parts of the resulting eigenvalues are the (real) eigenvalues of $\hat{\mathrm{h}} \cdot \overline{\mathrm{C}}$. The use of a real matrix is prompted by the existence of excellent methods to obtain the eigenvalues and eigenvectors. These procedures have not as yet been extended to the case of complex matrices. The eigenvalues are obtained by a QR transformation of $\hat{\mathrm{h}} \cdot \overline{\mathrm{C}}$ (refs. 41 to 43 ). The eigenvectors of the matrix are calculated from the Wielandt inverse iteration, as described by Wilkinson (ref. 43). These methods provide fast and accurate computations of the eigenvalues and eigenvectors of a general, real matrix.

The remaining portion of the field-dependent calculation is the final evaluation of the conductivity tensor, and its inversion to obtain the resistivity, as a function of magnetic field strength. 


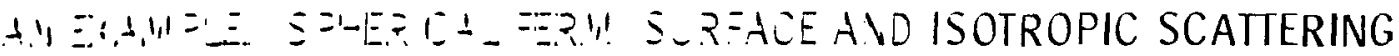

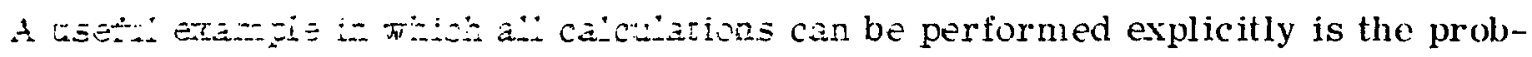
ler. of istrozic scarte-i-s in a free-electron model. For isotropic (spherically symmetric) scatterins. the $k \in r n e 1 \quad Q(\bar{k}, \bar{k})$ has the expansion

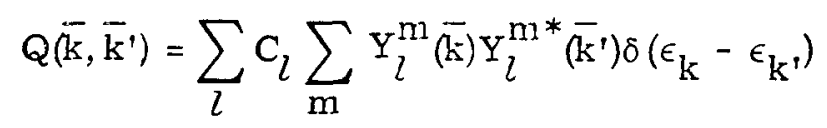

where the $\mathrm{Y}_{\ell}^{\mathrm{m}}$ are the normalized spherical harmonic functions.

The integrals over $\overline{\mathrm{k}}$-space may be transformed into integrals over the angles $\theta$ and $\varphi$ by the following considerations. The original definition of the volume element was

$$
\overline{\mathrm{dk}}=\frac{\mathrm{V}}{(2 \pi)^{3}} \mathrm{~d}^{3} \mathrm{k}
$$

From a transformation to spherical coordinates and the fact that $\epsilon=\hbar^{2} \mathrm{k}^{2} / 2 \mathrm{~m}$,

$$
\overline{\mathrm{dk}}=\frac{\mathrm{V}}{(2 \pi)^{3}} \frac{\mathrm{mk}}{\hbar^{2}} \mathrm{~d} \epsilon \mathrm{dS}
$$

where $d S=\sin \theta d \theta d \varphi$. Since each $\overline{\mathrm{k}}$ is restricted to the Fermi surface, the energy integration may be performed. Thus,

$$
\overline{\mathrm{dk}} \rightarrow \frac{\mathrm{V}}{(2 \pi)^{3}} \frac{\mathrm{mk}}{\hbar^{2}} \mathrm{dS} \equiv \mathrm{d} \Omega
$$

The scattering eigenvalue problem is solved with the aid of the orthogonality condition of the spherical harmonics,

$$
\int \mathrm{Y}_{l}^{\mathrm{m} *}(\mathrm{~S}) \mathrm{Y}_{l^{\prime}}^{\mathrm{m}^{\prime}}(\mathrm{S}) \mathrm{dS}=\delta_{l l^{\prime}} \delta_{\mathrm{mm}}
$$

The integration over the energy that is implicit in the operation $\mathrm{QY}_{l}^{\mathrm{m}}$ may be done immediately, from the preceding discussion, and thus, 


$$
\mathrm{QY}_{l}^{\mathrm{m}}=\int \mathrm{Q}\left(\overline{\mathrm{k}}, \overline{\mathrm{k}^{\prime}}\right) \mathrm{Y}_{l}^{\mathrm{m}} \overline{\left(\mathrm{k}^{\prime}\right) \mathrm{d} \Omega^{\prime}}=\frac{\mathrm{V}}{(2 \pi)^{3}} \frac{\mathrm{mk}^{3} \mathrm{~F}}{\hbar^{2}} \mathrm{C}_{l} \mathrm{Y}_{l}^{\mathrm{m}} \dot{\overline{\mathrm{k}})}
$$

where the point $\overrightarrow{\mathrm{k}}$ has coordinates $\left(\mathrm{k}_{\mathrm{F}}, \theta, \varphi\right)$. Then, also

$$
q(\bar{k})=\frac{v}{(2 \pi)^{3}} \frac{{ }^{m k}}{\hbar^{2}} C_{0}
$$

Thus, the scattering eigenvalue problem has the immediate solution

$$
\mathrm{QY}_{l}^{\mathrm{m}}=\alpha_{l} \mathrm{q} \overline{(\mathrm{k})} \mathrm{Y}_{l}^{\mathrm{m}} \overline{(\mathrm{k})}
$$

with $\alpha_{l}=\mathrm{C}_{l} / \mathrm{C}_{0}$. Each eigenvalue $\alpha_{l}$ is $(2 l+1)$-fold degenerate, corresponding to the $2 l+1$ functions $\left\{\mathrm{Y}_{l}^{\mathrm{m}}\right\}$ for each $l$. The $l^{\text {th }}$ irreducible representation of the rotation group has matrices of dimension $2 l+1$ and the $\mathrm{Y}_{l}^{\mathrm{m}}$ are the basis functions for the irreducible representation. The degeneracy of the scattering eigenvalues is due to the rotational symmetry of the scattering kernel, in the same manner as indicated for the point groups in the section SYMMETRY PROPERTIES OF THE SCATTERING EIGENFUNCTIONS.

The usefulness of this example lies primarily in the fact that the magnetic scattering eigenvalue problem can be solved analytically. The definition of the magnetic scattering problem was, from equation (24)

$$
\frac{\mathrm{im}}{\hbar}\left(\overline{\mathrm{v}}_{\mathrm{k}} \cdot \hat{\mathrm{h}} \times \nabla\right) \mathrm{b}(\overline{\mathrm{k}})=\tau(\mathrm{q}-\mathrm{Q}) \mathrm{b}(\overline{\mathrm{k}})
$$

The operator $Q$ commutes with the total angular momentum operator, and the term $\operatorname{im}\left(\bar{v}_{k} \cdot \hat{h} \times \nabla\right)$ is simply the component of the angular momentum in the direction of $\hat{h}$. Thus, the operator $T$ commutes with the total angular momentum, implying that the functions $\mathrm{b}$ are indexed by 2 . Further consideration shows that the magnetic eigenfunctions are simply the spherical harmonics $\mathrm{Y}_{l}^{\mathrm{m}}$. Transforming to a new set of spherical coordinates $\left(k_{F}, \Theta, \Phi\right)$, with the new polar axis along the direction of $\hat{h}$, results in the following simple form for equation (58):

$$
\mathrm{i} \frac{\mathrm{db}_{l}}{\mathrm{~d} \Phi}=\tau(\mathrm{q}-\mathrm{Q}) \mathrm{b}_{l}
$$


or,

$$
\mathfrak{i} \frac{\mathrm{d}}{\mathrm{d} \Phi} \mathrm{Y}_{l}^{\mathrm{m}}(\Theta, \Phi)=\tau(\mathrm{q}-\mathrm{Q}) \mathrm{Y}_{l}^{\mathrm{m}}(\Theta, \Phi)
$$

The new functions $\mathrm{Y}_{l}^{\mathrm{m}}(\Theta, \Phi)$ are linear combinations of the $\mathrm{Y}_{l}^{\mathrm{m}}(\theta, \varphi)$ with the same $l$-value. Thus $\mathrm{Y}_{l}^{\mathrm{m}}(\Theta, \Phi)$ is an eigenfunction of $\mathrm{Q}$ and

$$
\tau_{l}^{\mathrm{m}}=\frac{-\mathrm{m}}{\mathrm{q}\left(1-\alpha_{l}\right)}
$$

The relation between the scattering eigenvalue problem and the magnetic scattering problem is clear. The presence of the vector $\hat{h}$ in the operator $T$ removes the azimuthal degeneracy present in the scattering eigenfunctions.

Now that the magnetic eigenfunctions have been found analytically, we could proceed by using equation (26) for the conductivity. Although it is not necessary, it is illuminating to proceed with the calculation of the $\overline{\mathrm{C}}$ matrix. Because of the simple form of $\mathrm{T}$ in an $\hat{\mathrm{h}}$-fixed set of polar coordinates, it is easier to compute the matrix $\hat{\mathrm{h}} \cdot \overline{\mathrm{C}} \mathrm{di}-$ rectly. From equation (33),

$$
\left.\hat{\mathrm{h}} \cdot \overline{\mathrm{C}}=-\frac{\mathrm{im}}{\hbar}\left[\left(1-\alpha_{\ell^{\prime}}\right)\left(1-\alpha_{\ell^{\prime}}\right)\right]^{-1 / 2} \int \mathrm{Y}_{\ell}^{\mathrm{m}}(\mathrm{S}) \hat{(\hat{\mathrm{h}}} \cdot \overline{\mathrm{v}}_{\mathrm{k}} \times \nabla\right) \mathrm{Y}_{\ell^{\prime}}^{\mathrm{m}^{\prime}}(\mathrm{S}) \mathrm{d} \mathrm{S}
$$

where the symbol $S$ has been used for $(\theta, \varphi)$. Again, in terms of the variables $(\Theta, \Phi)$, the quantity $(\mathrm{m} / \hbar)\left(\hat{\mathrm{h}} \cdot \overline{\mathrm{v}}_{\mathrm{k}} \times \nabla\right)$ is simply $\mathrm{d} / \mathrm{d} \Phi$. The transformation from the spherical harmonics $\mathrm{Y}_{l}^{\mathrm{m}}(\mathrm{S})$ to the new coordinate system, resulting in $\mathrm{Y}_{l}^{\mathrm{m}}(\Theta, \Phi)$, is obtained from the fact that the $\mathrm{Y}_{l}^{\mathrm{m}}$ are basis functions for the irreducible representations of the rotation group. Thus, from reference 34 ,

$$
\mathrm{Y}_{l}^{\mathrm{m}}(\mathrm{S})=\sum_{\mathrm{m}^{\prime}} \mathrm{Y}_{l}^{\mathrm{m}^{\prime}}(\Theta, \Phi) \mathrm{D}_{\mathrm{mm}}^{(l)}(\hat{\mathrm{h}})
$$

a relation that is considered in more detail later in this section. Finally, we have

$$
(\hat{\mathrm{h}} \cdot \overline{\mathrm{C}})_{l \mathrm{~m}}^{l^{\prime} \mathrm{m}^{\prime}}=\left(1-\alpha_{l}\right)^{-1} \sum_{\mathrm{M}} \mathrm{MD}_{\mathrm{Mm}}^{(l)}(\hat{\mathrm{h}}) \mathrm{D}_{\mathrm{Mm}}^{(l)}(\hat{\mathrm{h}}) \delta_{l l^{\prime}}
$$


The major difference between the present example and the discussion of the section SYMMETRY PROPERTIES OF THE SCATTERING EIGENFUNCTIONS arises from the fact that different irreducible representations of the rotation group are not coupled in $\hat{\mathrm{h}} \cdot \overrightarrow{\mathrm{C}}$; the matrix is block diagonal in $l$. The symmetry breaking provided by the restriction to be a point group makes the structure of $\hat{h} \cdot \bar{C}$ more complicated.

Summarizing the magnetic scattering eigenvalue problem, the magnetic eigenvalues are

$$
\tau_{l}^{\mathrm{m}}=\frac{-\mathrm{m}}{\mathrm{q}\left(1-\alpha_{l}\right)} \quad \mathrm{m}=-l,-l+1, \ldots, ., l-1, l
$$

and the corresponding eigenfunctions are

$$
\mathrm{b}_{l \mathrm{~m}}(\overline{\mathrm{k}})=\mathrm{Y}_{l}^{\mathrm{m}}(\Theta, \Phi)=\sum_{\mathrm{m}^{\prime}} \mathrm{Y}_{l}^{\mathrm{m}^{\prime}}(\mathrm{S}) \mathrm{D}_{\mathrm{mm}}^{(\mathrm{l})}(\hat{\mathrm{h}})
$$

A comparison of equation (63) with equation (30) shows the correspondence between $\mathrm{D}_{\mathrm{mm}}^{2}$ and $\gamma_{2 \mathrm{n}}$.

The conductivity tensor is obtained from equation (26), with a factor of 2 included to count both spin states,

$$
\sigma_{\nu \mu}=2 \mathrm{e}^{2} \sum_{l, \mathrm{~m}} \frac{\beta_{\mu}^{l \mathrm{~m}}}{1-\mathrm{i} \omega \tau_{l}^{\mathrm{m}}} \int \mathrm{v}_{\nu} \mathrm{b}_{l \mathrm{~m}} \delta\left(\epsilon_{\mathrm{k}}-\epsilon_{\mathrm{F}}\right) \overline{\mathrm{dk}}
$$

where

$$
(q-Q)^{-1} v_{\mu}=\sum_{l m} \beta_{\mu}^{l m} b_{l m}
$$

to make the correspondence with equation (25). In the case of a spherical surface the velocity vector is everywhere radial, and

$$
m v_{\mu}=\hbar k_{\mu}=\hbar k_{F^{k}} \hat{k}_{\mu}
$$


or,

$$
\overline{\mathrm{v}}=\left(\frac{\mathrm{hk}_{\mathrm{F}}}{\mathrm{m}}\right) \hat{\mathrm{k}}
$$

defining the unit radius vector $\hat{\mathrm{k}}$, with components $\hat{\mathrm{k}}_{\mu}$. The $\hat{\mathrm{k}}_{\mu}$ have the property that they are linear combinations of only $l=1$ spherical harmonics. Thus, in both equations (64) and (65), the summations over $l$ are restricted to the terms for $l=1$. The final form for the conductivity is

$$
\sigma_{\nu \mu}=\frac{\mathrm{e}^{2} \mathrm{k}_{\mathrm{F}}^{2}}{\hbar} \frac{2 \mathrm{~V}}{(2 \pi)^{3}} \sum_{\mathrm{m}} \frac{\beta_{\mu}^{1 \mathrm{~m}}}{1-\mathrm{i} \omega \tau_{1}^{\mathrm{m}}} \int \hat{\mathrm{k}}_{\nu} \mathrm{b}_{1 \mathrm{~m}} \mathrm{dS}
$$

The factor in equation (66) that plays a role similar to the $g_{\nu \mathrm{n}}$ of equation (36) is the integral

$$
\mathrm{I}_{\nu \mathrm{m}}=\int \hat{\mathrm{k}}_{\nu} \mathrm{b}_{1 \mathrm{~m}}(\Theta, \Phi) \sin \theta \mathrm{d} \theta \mathrm{d} \varphi
$$

with $\mathrm{b}_{1 \mathrm{~m}}$ given in terms of the $\mathrm{Y}_{l}^{\mathrm{m}}(\mathrm{S})$ by equation (63). In order to evaluate these integrals, a specific representation for the D-matrix must be chosen. The $\mathrm{D}$ is the $3 \times 3$ (for $l=1$ ) rotation matrix that describes the rotation from the fixed coordinate system to the $h$-fixed coordinate system. By convention, this is a rotation matrix for the Euler angles $(\Phi, \Theta,-\Phi)$, and the form of $D$ is (ref. 34)

$$
D^{(1)}(\Phi, \Theta,-\Phi)=\left[\begin{array}{lll}
\frac{1}{2}(1+\cos \Theta) & \frac{1}{\sqrt{2}} \sin \Theta \mathrm{e}^{\mathrm{i} \Phi} & \frac{1}{2}(1-\cos \Theta) \mathrm{e}^{2 \mathrm{i} \Phi} \\
\frac{1}{\sqrt{2}} \sin \Theta \mathrm{e}^{-\mathrm{i} \Phi} & \cos \Theta & \frac{1}{\sqrt{2}} \sin \Theta \mathrm{e}^{\mathrm{i} \Phi} \\
\frac{1}{2}(1-\cos \Theta) \mathrm{e}^{-2 \mathrm{i} \Phi} & -\frac{1}{\sqrt{2}} \sin \Theta \mathrm{e}^{-\mathrm{i} \Phi} & \frac{1}{2}(1+\cos \Theta)
\end{array}\right]
$$


With this form for $\mathrm{D}$, the $\beta_{\mu}^{1 \mathrm{~m}}$ and the integrals $\mathrm{I}_{\nu \mathrm{m}}$ may be evaluated. The results are

$$
\begin{gathered}
\beta_{\mathrm{x}, \mathrm{y}}^{1 \mathrm{~m}}=\frac{\hbar \mathrm{k}_{\mathrm{F}}}{\mathrm{m}} \frac{1}{\mathrm{q}\left(1-\alpha_{1}\right)} \sqrt{\frac{2 \pi}{3}}\left[\mathrm{D}_{-1, \mathrm{~m}}^{(1) *}(\hat{\mathrm{h}}) \mp \mathrm{D}_{1, \mathrm{~m}}^{(1) *}(\hat{\mathrm{h}})\right] \\
\beta_{\mathrm{z}}^{1 \mathrm{~m}}=\frac{\hbar \mathrm{k}_{\mathrm{F}}}{\mathrm{m}} \frac{1}{\mathrm{q}\left(1-\alpha_{1}\right)} \sqrt{\frac{4 \pi}{3}}\left[\mathrm{D}_{0 \mathrm{~m}}^{(1) *}(\hat{\mathrm{h}})\right]
\end{gathered}
$$

and

$$
\begin{gathered}
\mathrm{I}_{\mathrm{xm}}=\sqrt{\frac{2 \pi}{3}}\left[\mathrm{D}_{-1, \mathrm{~m}}^{(1)}(\hat{\mathrm{h}})-\mathrm{D}_{1, \mathrm{~m}}^{(1)}(\hat{\mathrm{h}})\right] \\
\mathrm{I}_{\mathrm{ym}}=-\mathrm{i} \sqrt{\frac{2 \pi}{3}}\left[\mathrm{D}_{-1, \mathrm{~m}}^{(1)}(\hat{\mathrm{h}})+\mathrm{D}_{1, \mathrm{~m}}^{(1)}(\hat{\mathrm{h}})\right] \\
\mathrm{I}_{\mathrm{zm}}=\sqrt{\frac{4 \pi}{3}}\left[\mathrm{D}_{0 \mathrm{~m}}^{(1)}(\hat{\mathrm{h}})\right]
\end{gathered}
$$

The magnetic scattering eigenvalues for $l=1$ are obtained from equation (60) and, with the definition $\tau^{-1}=q\left(1-\alpha_{1}\right)$, are

$$
\begin{gathered}
\tau_{1}^{ \pm 1}=\mp \tau \\
\tau_{1}^{0}=0
\end{gathered}
$$

The conductivity may be evaluated directly, but the form of $\sigma$ itself is not of much interest. For example, from equation (66) the zz-component of $\sigma$ is 


$$
\sigma_{z \mathrm{z}}=\frac{\mathrm{e}^{2} \tau}{\mathrm{m}} \frac{2 \mathrm{~V}}{(2 \pi)^{3}} \frac{4 \pi}{3} \mathrm{k}_{\mathrm{F}}^{3}\left[\left|\mathrm{D}_{00}^{(1)}\right|^{2}+\frac{\left|\mathrm{D}_{10}^{(1)}\right|^{2}}{1-\mathrm{i} \omega \tau}+\frac{\left|\mathrm{D}_{-10}^{(1)}\right|^{2}}{1+\mathrm{i} \omega \tau}\right]=\frac{\mathrm{ne}^{2} \tau}{\mathrm{m}}\left[\cos ^{2} \Theta+\frac{\sin ^{2} \Theta}{1+(\omega \tau)^{2}}\right]
$$

where

$$
\mathrm{n}=\frac{2 \mathrm{~V}}{(2 \pi)^{3}} \frac{4 \pi}{3} \mathrm{k}_{\mathrm{F}}^{3}
$$

is the density of electrons in the metal.

The more interesting result, the quantity that would be measured experimentally, is the resistivity tensor $\rho_{\nu_{\mu}}=\left(\sigma^{-1}\right)_{\nu_{\mu}}$. With the definitions of $\gamma=\omega \tau$ and $\rho_{0}=\mathrm{m} / \mathrm{ne}^{2} \tau$, the resistivity tensor is

$$
\frac{\rho(\gamma)}{\rho_{0}}=\left[\begin{array}{lll}
1 & -\gamma \cos \Theta & \gamma \sin \Theta \sin \Phi \\
\gamma \cos \Theta & 1 & -\gamma \sin \Theta \cos \Phi \\
-\gamma \sin \Theta \sin \Phi & \gamma \sin \Theta \cos \Phi & 1
\end{array}\right]
$$

This result is the familiar free-electron result, with the difference that the coordinate system has been rotated to axes in which the magnetic field is in the direction $(\Theta, \Phi)$.

If the model of a spherical Fermi surface is to have any validity for the alkali metals, in particular potassium, it is clear that a spherically symmetric scattering model is not sufficient to explain the experimental results (ref. 44). One possible model for the scattering, the degenerate kernel model, is the subject of the next section.

\section{DEGENERATE KERNEL THEORY}

The Boltzmann equation (BE) provides the framework for the description of those transport properties of metals that do not arise from strictly quantum-mechanical effects such as the Landau level quantization. The nature of the BE, an integrodifferential equation for the distribution function, has always hampered attempts to provide calculations for any but the simplest models for metals. The difficult part of any calculation has always been the manner in which the scattering term of the BE has been treated. 
The relaxation-time approximation (the assumption of either a constant or a scalar function of wave number to replace the effect of the scattering) has been the most common approximation. This achieves the desired reduction of the BE, but at the cost of averaging the scattering over all states. Thus, the knowledge about the relation between the topology of the Fermi surface and the galvanomagnetic properties of the metal has been gained at the expense of an understanding of the electron's interaction with its environment in the metal.

Sondheimer (ref. 21) formulated a way to treat the scattering in a more complete manner by making the ad hoc assumption that the kernel, $Q\left(\bar{k}, \bar{k}^{\prime}\right)$ of equation (18), is degenerate. Written explicitly, this assumption is that

$$
Q\left(\bar{k}, \bar{k}^{\prime}\right)=\sum_{\substack{i, j=1}}^{N} b_{i j} q_{i}(\bar{k}) q_{j}\left(\bar{k}^{\prime}\right)
$$

where $\left\{q_{\mathbf{i}}\right\}$ is a set of $\mathrm{N}$ arbitrary functions of integrable square (ref. 24). The impetus for this assumption is the existence of well-documented methods of treating Fredholm integral equations having degenerate kernels (ref. 22). Since the summation in equation (67) is over a finite number of terms, the integral equation reduces to a set of linear equations.

The approximation of the scattering kernel by a degenerate kernel may be partly justified as a scattering model. Further, the basis functions $q_{i}$ are not arbitrary. Mann (refs. 25 to 27) has demonstrated that, for an electron gas that interacts with a static system of impurities, the transition probability for scattering could be written as in equation (67), where the $\left\{q_{i}\right\}$ are Wannier functions for the electrons. Further work by Mann (ref. 27) and Seeger et al. (ref. 45) discusses the transport properties of such a system as a model for a metal, but as yet no treatment for the galvanomagnetic properties of the model has appeared.

In this section the galvanomagentic properties that result from a spherical Fermi surface and a simple degenerate kernel are discussed. This discussion serves three functions. First, an inconsistency in the Jones and Sondheimer calculation (ref. 24) is examined and its origin in the arbitrary truncation of the degenerate kernel is demonstrated. Second, the natural representation provided by the symmetry-adapted functions becomes even more apparent when applied to a degenerate kernel. Last, the sample calculations show the strong effect upon the galvanomagnetic properties for a simple anisotropic scattering model, for even a spherical Fermi surface.

It should be apparent from the discussion of scattering eigenfunctions, and in particular the appearance of equation (45), that the degenerate kernel theory has even wider applicability than has been realized previously. With the assumption of the symmetry 
of the kernel with respect to the point group operations, that is,

$$
\mathrm{Q}\left(\mathrm{R} \overline{\mathrm{k}}, \mathbf{R} \overline{\mathrm{k}}^{\dagger}\right)=\mathrm{Q}\left(\overline{\mathrm{k}}, \overline{\mathrm{k}} \overline{\mathrm{k}}^{p}\right)
$$

there exists a biharmonic expansion of the function $Q$ in the symmetry-adapted functions. If this expansion is truncated after $\mathrm{N}$ terms, then the result is equivalent to equation (67). The advantage of this formulation is that the integrals of the symmetryadapted functions may easily be calculated from the representation theory of the point group, as has been demonstrated here. The main disadvantage of working with a degenerate kernel lies in the uncertain relation between the postulated kernel and the actual scattering processes. To make this connection satisfactorily requires the calculation of $\mathrm{Q}\left(\overline{\mathrm{k}}, \overline{\mathrm{k}}^{\prime}\right)$ from an interaction Hamiltonian, and then the use of the orthogonality properties of the symmetry-adapted functions to invert equation (45) for the expansion coefficients $\mathrm{A}_{l l}^{\mathrm{j}}$, . This inversion adds a substantial amount of labor to the computations labor that is unnecessary in view of the present formalism which only requires the function $Q$.

To return again to the work of Jones and Sondheimer, the use of the $\left\{q_{i}\right\}$ as basis functions allows a solution to the linearized BE for the conductivity and, hence, the resistivity. Since the basis functions are unspecified, the resulting expressions are formal in nature, equivalent to equation (23). To make possible a comparison between the results of reference 24 and the present work, consider the model consisting of a spherical Fermi surface and the scattering kernel

$$
\begin{array}{r}
\mathrm{Q}\left(\overline{\mathrm{k}}, \overline{\mathrm{k}^{\prime}}\right)=\mathrm{a}_{0}+\sum_{\alpha}\left[\mathrm{c}_{2} \mathrm{k}_{\alpha} \mathrm{k}_{\alpha}^{\prime}+\mathrm{a}_{4}\left(\mathrm{k}_{\alpha}^{4}+\mathrm{k}_{\alpha}^{\prime 4}\right)+\mathrm{b}_{4} \mathrm{k}_{\alpha}^{2} \mathrm{k}_{\alpha}^{\prime 2}+\mathrm{c}_{4}\left(\mathrm{k}_{\alpha} \mathrm{k}_{\alpha}^{\prime}{ }^{3}+\mathrm{k}_{\alpha}^{3} \mathrm{k}_{\alpha}^{\prime}\right)\right. \\
+\sum_{\alpha \neq \beta} \mathrm{e}_{4} \mathrm{k}_{\alpha} \mathrm{k}_{\beta} \mathrm{k}_{\alpha}^{\prime} \mathrm{k}_{\beta}^{\prime}
\end{array}
$$

as given by equation (3.4) of reference 24. The constants $a_{0}, a_{4}, b_{4}, c_{2}, c_{4}$, and $e_{4}$ must be chosen consistent with the requirements that $Q\left(\bar{k}, \bar{k}^{\prime}\right)$ be normalized and positive-definite for all $\overline{\mathrm{k}}$ and $\overline{\mathrm{k}^{\prime}}$. In terms of these partially adjustable parameters, Jones and Sondheimer show in their figures 1 to 3 (ref. 24) the variation of the longitudinal magnetoresistance as the magnetic field varies from $\langle 100\rangle$ to $\langle 111\rangle$. More important than the quantitative results is the observation that the behavior is not that of a free-electron gas. There is a nonzero longitudinal magnetoresistance that initially increases but saturates as the magnetic field becomes large. Thus, a reasonably simple anisotropy in the scattering kernel produces a marked effect in the galvanomagnetic properties, even for spherical Fermi surfaces. 
Rather than simply taking the kernel of equation (68) with some coefficients chosen for comparison with those of reference 24, consider in a little more detail the problems involved in working with a degenerate kernel. This is done by using equation (68) as a working example. It is easily seen that most of equation (68) is extraneous. Jones and Sondheimer show that only that part of $Q$ which is odd under coordinate inversion can contribute to the transport current. They use this property to eliminate the problem of the singularity that is in the operator $q-Q$. This report shows that a condition stronger than the parity requirement may be formulated. Of all the irreducible representations contained in $\mathrm{Q}$, only those which are not excluded by the Kronecker product selection rules for the formation of the $\bar{C}$ matrix need be considered. Since, for the cubic group, this excludes all irreducible representations with even parity, the condition of Jones and Sondheimer (ref. 24) is included.

All these observations may be illustrated by considering as an example the kernel

$$
\mathrm{Q}\left(\overline{\mathrm{k}}, \overline{\mathrm{k}}^{\prime}\right)=\frac{1}{(4 \pi)^{2}}+\sum_{\mathrm{r}=1}^{3} \sum_{\substack{l^{\prime}=1,3 \\ l^{\prime}=1,3}} \mathrm{~A}_{l l^{\prime}} \Gamma_{15_{\mathrm{x}}} \Gamma_{15}, \mathrm{r}_{(\overline{\mathrm{k}}) \mathrm{x}_{l^{\prime}}} \Gamma_{15}, \mathrm{r}_{\left(\overline{\mathrm{k}^{\prime}}\right)}
$$

The constant term serves only to normalize $\iint \mathrm{Q}\left(\overline{\mathrm{k}}, \overline{\mathrm{k}}^{\prime}\right) \overline{\mathrm{dk}} \overline{\mathrm{dk}}$, and is not important otherwise. The function $q(\bar{k})$, obtained by integrating equation (69) over $\overline{\mathrm{dk}^{\prime}}$ is simply $1 / 4 \pi$. This kernel differs from equation (68) in two respects, one minor and one quite substantial. The quartic factors multiplying $\mathrm{a}_{4}$ and $\mathrm{b}_{4}$ of equation (68) give integrals that do not vanish when $q(\bar{k})$ is formed. This minor inconvenience is due to the use of nonorthogonal basis functions. This restriction of attention to a constant $q(\bar{k})$ is of only minor consequence. The major difference can be seen by a consideration of the criteria for the choice of the coefficients, in both equations (68) and (69). The condition that must be satisfied, once the form of $Q$ is chosen to satisfy the group symmetry requirements, is that $Q$ must be positive-definite. In the Jones and Sondheimer discussion (ref. 24) of the degenerate kernel, this meant the evaluation of $Q$ for sufficient values of $\overline{\mathrm{k}}$ and $\overline{\mathrm{k}}$ ' to give inequalities that must be satisfied by the coefficients. Equation (49) implies that the scattering eigenvalues are obtained from a secular determinant of the $A_{\imath l}$, of equation (69). (The difference between $Q$ and the reduced kernel $\mathrm{Q}\left(\overline{\mathrm{k}}, \overrightarrow{\mathrm{k}}^{\prime}\right) / \sqrt{\mathrm{q}(\overline{\mathrm{k}}) q\left(\overline{\left.\mathrm{k}^{\prime}\right)}\right.}$ is a constant multiplier, so that eq. (69) also represents the reduced kernel.)

Explicity, since the matrix $A$ is Hermitian (real symmetric when the real representaions for the Kubic harmonics are used), the secular equation is 


$$
\left|\begin{array}{ll}
\mathrm{A}_{11}-\alpha & \mathrm{A}_{13} \\
\mathrm{~A}_{13} & \mathrm{~A}_{33}-\alpha
\end{array}\right|=0
$$

At this point, the major distinction between the two kernels (eqs. (68) and (69)) is apparent. The coefficients of $A_{33}$, arising from $l=3$ spherical harmonics, are of the sixth degree in $\overline{\mathrm{k}}$ and $\overline{\mathrm{k}}^{\prime}$. The Jones and Sondheimer kernel was truncated to include terms only to the fourth degree. This omission of the $A_{33}$ term from equation (68) has interesting consequences, since it implies that the scattering operator cannot be positivedefinite. This observation may be made from equation (70) by setting $\mathrm{A}_{33}=0$ and, thus, for any nonzero $A_{11}$ and $A_{13}$ there is one root that must be negative. Since a necessary and sufficient condition for an operator to be positive-definite includes the requirement that all eigenvalues be positive (ref. 23), the truncation of the series must be performed carefully.

The galvanomagnetic properties of a system with a spherical Fermi suriace and a scattering kernel of the form given by equation (69) are easily calculated with the present formalism. This discussion is not meant to provide a comparison with any experiment, but, rather, is an investigation of the properties of a simple scattering kernel. The method that was chosen to accomplish this investigation was to choose a value for $A_{11}$ and $\mathrm{A}_{33}$ (in this case the values chosen were 0.5 and 0.25 , respectively) and to determine the range of $\mathrm{A}_{13}$ that gave positive, nonzero eigenvalues $\alpha$. The solution of equation (70) for the eigenvalues and the eigenvectors, providing the appropriate coefficients for the linear combinations of the $\mathrm{x}_{1}$ and $\mathrm{x}_{3}$ to form the scattering eigenfunctions, then provides sufficient information to compute the resistivity tensor as a function of the magnetic field strength and orientation.

A convenient way to present the results of the computations is to define a quantity that parametrizes the strength of the mixing term $A_{13}$. One such parameter is the ratio of the off-diagonal part to the Euclidean norm of the matrix

$$
\left(\sum_{i, j} A_{i j}^{2}\right)^{1 / 2}
$$

The coefficients are then normalized to give a unit norm, so that the quantity $\mathrm{A}_{13}$ is itself the desired anisotropy parameter. The components of the resistivity tensor that are of interest are the longitudinal resistance $\rho_{\mathrm{zz}}$, the Hall resistance $\rho_{\mathrm{xy}}$, and the average transverse resistance $\left.\rho_{\mathrm{tr}}=\rho_{\mathrm{xx}}+\rho_{\mathrm{yy}}\right) / 2$. These quantities are calculated for different values of $A_{13}$, as functions of $H$, and for different field orientations $\hat{h}$. 
The values chosen are intended to be representative of both a weak-scattering case $\left(A_{13}=0.06275\right)$ and a strong-scattering case $\left(A_{13}=0.26726\right)$. This characterization of "weak" and "strong" is justified by the extreme dependence of the saturation magnetoresistance values upon the anisotropy parameter. The constant $\mathrm{q}^{-1}$ is used as an effective relaxation time $\tau_{\text {eff, }}$, or simply $\tau$, in agreement with the usual experimental convention. Figures 1 (a) and 2(a) show the change in longitudinal and transverse resistance as a function of $\omega \tau$, for fields in the two symmetry directions, $\langle 100\rangle$ and $\langle 111\rangle$. Figures $1(\mathrm{~b})$ and $2(\mathrm{~b})$ give the Hall resistance $\rho_{\mathrm{xy}}$ and the Hall constant $\left(\mathrm{d} \rho_{\mathrm{xy}} / \mathrm{d}(\omega \tau)\right)$, for the applied field along the $\langle 111\rangle$ direction. The pairs of curves for each value of $\mathrm{A}_{13}$ are qualitatively the same, but the scale has been changed for the larger $\mathrm{A}_{13}$, demonstrating the strong dependence upon the scattering. The magnetoresistance curves show the initial rise (probably quadratic) and ultimate saturation that are expected for a spherical Fermi surface model (ref. 3). The Hall properties reflect this low-field behavior. Although the Hall resistance $\rho_{x y}$ is predominantly linear, there is a small region near $\mathrm{H}=0$ that shows a nonlinear behavior. This is indicated by the variation of the Hall constant in figures $1(b)$ and $2(b)$. The primary difference between the two scattering cases lies in the scale of the effects. As the off-diagonal part, $A_{13}$, increases, the nonlinear behavior near the origin becomes more pronounced, saturation of the magnetoresistance requires a larger field, and the value of the saturation magnetoresistance increases quite markedly.

To complete the discussion of this scattering model, figures $3(\mathrm{a})$ and (b) show the variation in the saturation values of $\rho_{\mathrm{zz}}$ and $\rho_{\mathrm{tr}}$ as a function of field orientation around the triangle $(\langle 100\rangle,\langle 111\rangle,\langle 110\rangle,\langle 100\rangle)$.

Although the simplified, degenerate kernel model discussed is much too simplified to be a serious model for a metal, the discussion of the present section shows some points that hear emphasizing. There is a great deal of information contained in the field-independent quantities $\overline{\mathrm{C}}$ and $\mathrm{g}_{\nu \mathrm{n}}$. The evaluation of all components of the resistivity tensor as a function of field strength and orientation is a simple task, and the amount of information that can be obtained about the galvanomagnetic properties of the system should be apparent at this point. This complete description of the $\rho$ tensor must be compared with previous calculations (ref. 24) that must, perforce, concentrate upon only one component.

In summary, the degenerate kernal model clearly demonstrates the effect of a simple scattering model upon the galvanomagnetic properties of an electronic system with a spherical Fermi surface. Even the simple model discussed here gives results markedly different from free-electron behavior. This simplified model is not suggested as a realistic model for potassium, the metal it might be thought most nearly to represent. On the other hand, the discussion of even this simple model demonstrates the power and usefulness of a method capable of treating the electron scattering. 


\section{CONCLUSIONS}

The independent-particle model for conduction electrons in metals and the Boltzmann equation have provided a great deal of information about conduction processes in metals. Such diverse effects as magnetoresistance, cyclotron resonance, and magneto-acoustic attenuation (among many others) have been explained, at least qualitatively, with the semiclassical theory (refs. 3 and 16). Even the descriptions of quantum-mechanical effects, such as the de Haas - van Alphen effect, have used the concepts provided by the semiclassical theory as a basis (ref. 2). The current state of experiments on metals indicates that the qualitative description provided by the relaxation-time approximation is no longer sufficient.

If galvanomagnetic experiments are to be used to probe the interactions of the conduction electrons with the environment in the metal, then clearly a theory adequate to support these experiments must be more detailed than any theory is at the present. The ideal theory would allow the inverting of galvanomagnetic data to obtain the scattering

probability $Q\left(\bar{k}, \bar{k}^{\prime}\right)$. This goal cannot be completely attained, but this report provides a method to partly fill the needs. The scattering probability is used in a procedure that gives a closed-form solution to the Boltzmann equation for a metal in static, homogeneous, electric and magnetic fields.

From a model for the Fermi surface and the scattering processes, the resistivity tensor may be calculated for any magnetic field strength or orientation with respect to the crystal axes. A feature of this formalism is the ability to separate geometrical effects caused by the Fermi surface topology from anisotropic scattering effects. For a large number of metals the Fermi surface has been very accurately determined from band structure calculations, supported by experiment. Using this information as input to the procedure makes possible quantitative judgments about the effect of the scattering. The example of the degenerate kernal model discussed in the preceding section demonstrates the strong role that the scattering plays in the galvanomagnetic properties even for a metal with the simplest Fermi surface, a sphere. The vector mean free path $\bar{\Lambda}(\bar{k})$ has been introduced (refs. 8 and 9 ) to treat as completely as possible the anisotropies inherent in the system.

The formalism discussed in this report has been limited to the solution of the Boltzmann equation describing a metal in static electric and magnetic fields, for the case of elastic scattering. As discussed in the text, this limitation upon the scattering is not stringent; the formalism may be generalized to include inelastic scattering, at a cost of a substantial increase in the numerical work that implements the formalism. In addition, the linearization procedure and the method of solution of the BE should provide a valuable method of attack for other transport phenomena. Following the discussion of Taylor (refs. 8 and 9) for the thermopower of copper, this procedure is applicable to a 
description of thermomagnetic effects in metals, currently a subject of substantial experimental interest.

In summary, a formal solution to the linearized Boltzmann equation, valid for any strength of the magnetic field, has been presented. The numerical procedure to apply this formal solution to any Fermi surface to investigate the scattering processes in a metal has been described. As an example of the power of this technique, some consequences of a scattering model introduced by Sondheimer (ref. 21) for a spherical Fermi surface model have been investigated.

\section{Lewis Research Center,}

National Aeronautics and Space Administration, Cleveland, Ohio, September 8, 1969, 129-02. 


\section{APPENDIX A}

\section{SCATTERING AND MAGNETIC SCATTERING EIGENVALUE PROBLEM}

In the course of solving the linearized Boltzmann equation it is necessary to consider the eigenvalues and eigenfunctions of two non-Hermitian operators. In addition to the existence of such eigenvalues it is also necessary to assume that these eigenfunctions form a complete set for the expansion of functions within a Brillouin zone. The two problems are, in fact, quite similar, and it is instructive to consider the simpler case of the field-free scattering eigenfunctions first.

The integral operator $Q$ is a real, bounded, positive-definite linear operator (ref. 23), and as such possesses a real eigenvalue spectrum. It is not necessary to assume that the kernel $\mathrm{Q}\left(\overline{\mathrm{k}}, \overline{\mathrm{k}}^{\prime}\right)$ is degenerate, so that, in general, the number of eigenvalues is denumerably infinite, but bounded. Unfortunately, the operator which appears in the solution of the Boltzmann equation, as evidenced in equation (28), is $q^{-1} Q$, which will not be Hermitian unless $q$ is constant. Instead of this operator, the following eigenvalue problem can be considered

$$
\int \frac{\mathrm{Q}\left(\overline{\mathrm{k}}, \overline{\mathrm{k}^{\prime}}\right)}{\sqrt{\mathrm{q}(\overline{\mathrm{k}}) \mathrm{q}\left(\overline{\mathrm{k}^{\prime}}\right)}} \mathrm{Y}_{\mathrm{n}}\left(\overline{\mathrm{k}^{\prime}}\right) \mathrm{d} \Omega^{\prime}=\alpha_{\mathrm{n}} \mathrm{Y}_{\mathrm{n}}(\overline{\mathrm{k}})
$$

Since $\mathrm{Q}\left(\overline{\mathrm{k}}, \overline{\mathrm{k}}^{\dagger}\right)$ is a probability function, the integrated kernel $\mathrm{q}$ is a real function of $\overline{\mathrm{k}}$ and will not vanish unless $Q\left(\overline{\mathrm{k}}, \overline{\mathrm{k}}^{\prime}\right)$ is identically zero for $\overline{\mathrm{k}}$ and all $\overline{\mathrm{k}}$. The kernel of equation (A1) is symmetric, real, and continuous, so that the existence of the eigenvalues $\alpha_{n}$ and the eigenfunctions $Y_{n}(\bar{k})$ are guaranteed.

Now define a new set of functions $a_{n}(\bar{k})$ such that

$$
\mathrm{Y}_{\mathrm{n}} \overline{(\mathrm{k})}=\mathrm{a}_{\mathrm{n}}(\overline{\mathrm{k}}) \sqrt{\mathrm{q}(\overline{\mathrm{k}})}
$$

Then equation (A1) may be written as

$$
\mathrm{q}^{-1}(\overline{\mathrm{k}}) / \mathrm{Q}\left(\overline{\mathrm{k}}, \overline{\mathrm{k}}^{\prime}\right) \mathrm{a}_{\mathrm{n}}\left(\overline{\mathrm{k}^{\prime}}\right) \mathrm{d} \Omega^{\prime}=\alpha_{\mathrm{n}} \mathrm{a}_{\mathrm{n}}(\overline{\mathrm{k}})
$$

which is just the eigenvalue problem for the operator $q^{-1} Q$. Thus, the eigenvalues of $\mathrm{q}^{-1} \mathrm{Q}$ are identical to the eigenvalues of equation (A1), and the eigenfunctions are obtained by inverting equation (A2). 
The orthogonality condition for the scattering eigenfunctions (eq. (29)) follows from the symmetry of the kernel of equation (A1). By writing equation (A1) for another eigenfunction (e.g., $\mathrm{Y}_{\mathbf{r}}\left(\overline{\left.\mathrm{k}^{\prime}\right)}\right)$ and using the symmetry of the kernel, the result is

$$
\left(\alpha_{\mathrm{r}}-\alpha_{\mathrm{n}}\right) \int \mathrm{Y}_{\mathrm{r}} \overline{(\mathrm{k}) \mathrm{Y}_{\mathrm{n}}} \overline{(\mathrm{k})} \mathrm{d} \Omega=0
$$

Thus, for $\alpha_{\mathbf{n}} \neq \alpha_{\mathbf{r}}$, the desired orthogonality is attained. If, on the other hand, $\alpha_{\mathrm{n}}=\alpha_{\mathrm{r}}$, the degenerate eigenfunctions may be made orthogonal by the Gram-Schmidt. orthogonalization process (ref. 23). From this relation then, equation (33) follows immediately by the substitution of equation (A2), that is,

$$
\int a_{r}(\bar{k}) q(\bar{k}) a_{n}(\bar{k}) d \Omega=\delta_{r n}
$$

The completeness of the scattering eigenfunctions $a_{n}$ can also be seen from equation (A2). The $Y_{n}(\bar{k})$, being an orthogonal set of eigenfunctions of a Hermitian operator, form a complete set of functions. This means that, given a function $f(\bar{k})$, there exists a set of coefficients $C_{n}$ such that

$$
f(\bar{k})=\sum_{n} C_{n} Y_{n}(\bar{k})
$$

Since, from equation (A2), each $a_{n}$ is obtained from the corresponding $Y_{n}$ by division by the function $q(\bar{k})$ for all $n$, then

$$
\frac{f(\bar{k})}{q(\bar{k})}=\sum_{n} C_{n} a_{n}(\bar{k})
$$

in which the coefficients $C_{n}$ are the same as in equation (A4). Equivalently, the expansion

$$
g(\bar{k})=\sum_{n} D_{n} a_{n}(\bar{k})
$$

may be inverted to obtain the $D_{n}$ by multiplying by the product $a_{r} q$ and using equation (A3), that is, 


$$
D_{r}=\int g(\bar{k}) q(\bar{k}) a_{r}(\bar{k}) d \Omega
$$

which is another way of describing completeness.

This completes the discussion of the existence and completeness of the fieldindependent scattering eigenfunctions. Not only is this discussion important in its own right; it is useful in guiding the treatment of the magnetic scattering eigenvalue problem. The problem involved here lies in the fact that the magnetic scattering operator $\mathrm{T}$, although it is the product of two Hermitian operators, is not itself Hermitian, in general. Consider a more general problem and write an operator $\mathbf{T}$ as the product of two operators

$$
T=Q^{-1} P
$$

where $\mathbf{P}$ and $\mathrm{Q}$ (and thus $\mathrm{Q}^{-1}$ ) are each Hermitian. The operator $\mathrm{T}$ will not be Hermitian unless $P$ and $Q^{-1}$ commute. There is the additional assumption that $Q$ is a positive-definite, bounded, linear operator. With this assumption the formal definition of the square-root operator $Q^{1 / 2}$ is possible (ref. 46).

Following the discussion of the scattering operator, define a new operator ' $T$ ' as the product

$$
\mathrm{T}^{\prime}=\mathrm{Q}^{-1 / 2} \mathrm{PQ}^{-1 / 2}
$$

Since $T^{\prime}$ is Hermitian, the purpose of the construction, it has eigenvalues and eigenfunctions defined by the equation

$$
\mathrm{T}^{\prime} \mathrm{x}_{\mathrm{n}}=\mathrm{Q}^{-1 / 2} \mathrm{PQ}^{-1 / 2} \mathrm{x}_{\mathrm{n}}=\tau_{\mathrm{n}} \mathrm{x}_{\mathrm{n}}
$$

Now, operating on the left side of equation (A9) with $\mathrm{Q}^{-1 / 2}$ results in

$$
\mathrm{Q}^{-1} \mathrm{P}\left(\mathrm{Q}^{-1 / 2} \mathrm{x}_{\mathrm{n}}\right)=\tau_{\mathrm{n}}\left(\mathrm{Q}^{-1 / 2} \mathrm{x}_{\mathrm{n}}\right)
$$

Thus, a comparison of equation (A10) with (A7) shows that the eigenvalues of $T$ are identical with the eigenvalues of $\mathrm{T}^{\dagger}$ (and as a consequence must also be real) and the eigenfunctions of $T$ are the eigenfunctions of $T^{\prime}$ modified by the operator $Q^{-1 / 2}$, or

$$
b_{n}(\bar{k})=Q^{-1 / 2} x_{n}(\bar{k})
$$


Since the $x_{n}$ form a complete set, the same arguments as presented before indicate that the $b_{n}$ are also complete.

This discussion applies to the magnetic scattering problem by making the associations:

$$
\begin{gathered}
\mathrm{Q} \rightarrow(\mathrm{q}-\mathrm{Q}) \\
\mathrm{P} \rightarrow \frac{\mathrm{im}}{\hbar}\left(\overline{\mathrm{v}}_{\mathrm{k}} \cdot \hat{\mathrm{h}} \times \nabla\right)
\end{gathered}
$$

Although this discussion is instructive when applied to the magnetic scattering operator, it cannot be considered rigorous. The difficulty lies in the fact that the operator $P$, being by association a derivative operator, is not bounded. There is no guarantee that the eigenfunctions of $T$ lie in the space spanned by the scattering eigenfunctions $a_{n}(\vec{k})$. The question then is the existence of the expansion (eq. (34)) for the $\mathrm{b}_{l}$ in terms of the $a_{n}$. The ultimate justification for the use of this expansion is that solutions for the expansion coefficients $\gamma_{\ell n}$ can be obtained through the solution of equation (34). 


\section{APPENDIX B}

\section{PROOF OF GROUP THEORETIC THEOREMS AND RESULTS}

This appendix concerns itself with the theory of group representations and the properties of the scattering eigenfunctions of equation (28). Before starting this topic, it will be useful to expand upon the discussion of the basis functions of irreducible representations presented in the main text.

The configuration space used here is the set of all points on the Fermi surface, which is considered to be a single sheet with a single-valued position vector $\overline{\mathrm{k}}$ at each point. Under the action of a group $\mathscr{G}$, two points labeled $\overline{\mathrm{k}}$ and $\overline{\mathrm{k}}$ are equivalent if $\overline{\mathrm{k}}^{\prime}=\mathrm{R} \overline{\mathrm{k}}$, where $\mathrm{R}$ is a $3 \times 3$ matrix representation of a group operation $\mathscr{R}$ of $\mathscr{G}$. If the group is of order $\mathrm{g}$, then a point $\overline{\mathrm{k}}$ will have $\mathrm{g}$ equivalent points (or images) on the surface, under $\mathscr{G}$. Not all $\overline{\mathrm{k}}$ will have $\mathrm{g}$ distinct images, and these points are usually referred to as "high-symmetry" points.

The principal problem of interest is the effect of the group operation $\mathscr{R}$ upon a function of $\bar{k}$. The general definition of this action is (ref. 34)

$$
\mathscr{R} \mathrm{f}\left(\overline{\mathrm{k}^{\imath}}\right)=\mathrm{f}(\overline{\mathrm{k}})
$$

where, again, $\overline{\mathrm{k}}^{\prime}=\mathrm{R} \overline{\mathrm{k}}$. The notation here is slightly deceptive. The left side of equation (B1) means that the value of the function $\mathscr{R} \mathrm{f}$, whatever that is, when evaluated at the point $\overline{\mathrm{k}}^{\prime}$, is equal to the value of the function $f$ at the point $\bar{k}$.

The partners of a set of basis functions are defined by the equation

$$
\mathscr{R} \psi_{\mu}^{\alpha} \overline{(\mathrm{k})}=\sum_{\lambda=1}^{\mathrm{n}_{\alpha}} \mathrm{D}_{\lambda \mu}^{(\alpha)}(\mathrm{R}) \psi_{\lambda}^{\alpha}(\overline{\mathrm{k}})
$$

This equation means that, given the $\alpha^{\text {th }}$ irreducible representation of $\mathscr{G}$, there is a set of $\mathrm{n}_{\alpha}$ functions, where $\mathrm{n}_{\alpha}$ is the dimension of $\alpha$, such that a transformed function $\mathscr{R} \psi_{\mu}$, evaluated at the point $\mathrm{k}$, is a linear combination of all the basis functions. These functions are not uniquely determined until all the matrices of the irreducible representations, the $\mathrm{D}_{\lambda \mu}^{(\alpha)}(\mathrm{R})$, are explicitly stated. All that is guaranteed is that such a basis set exists for all $\alpha^{\prime}$ of $\mathscr{G}$, and that there is a prescription for obtaining the functions, given the $\mathrm{D}$ matrices.

The inverse of the group operation $\mathscr{R}$ must be in $\mathscr{G}$, so that equation (B1) may be inverted to obtain 


$$
\mathrm{f}\left(\overline{\mathrm{k}^{\prime}}\right)=\mathscr{R}^{-1} \mathrm{f}(\overline{\mathrm{k}})
$$

Thus, the basis function, evaluated at an image point is

$$
\psi_{\mu}^{\alpha} \overline{\left(\mathrm{k}^{\prime}\right)}=\sum_{\lambda} \mathrm{D}_{\mu \lambda}^{(\alpha)^{*}}(\mathrm{R}) \psi_{\lambda}^{\alpha} \overline{(\mathrm{k})}
$$

since the irreducible representation may always be chosen to be unitary. The relation, of fundamental importance in the material to follow, relates the basis function at an image point to the set of basis functions evaluated at the generating point.

It was discussed briefly in the main text that it is possible to form linear combinations of the spherical harmonics for a given $l$ to form basis functions for some irreducible representation of a point group $\mathscr{G}$. The point of importance is that an infinite set of such linear combinations, in other words for all $l$, forms an orthonormal complete set of functions, spanning the same space of functions as do the spherical harmonics. The full discussion of the relation between the symmetry-adapted functions and the spherical harmonics is well documented in the literature (e.g., the excellent article by Altmann and Cracknell (ref. 37) and the references quoted therein) and is beyond the scope of the work being described here. Let it suffice to say that there exists such a set of functions, characterized by $\mathrm{x}_{l}^{\mathrm{jr}}(\overline{\mathrm{k}})$, where

$$
\mathrm{x}_{l}^{\mathrm{j} \mathrm{r}} \overline{\left(\mathrm{k}^{\prime}\right)}=\sum_{\mathrm{s}} \mathrm{x}_{l}^{\mathrm{js}} \overline{(\mathrm{k})} \mathrm{D}_{\mathrm{rs}}^{\mathrm{j} *}(\mathrm{R})
$$

The appropriate orthonormality condition is

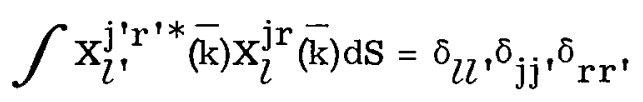

The notation used, as described in the main text, is that $\mathrm{x}_{l}^{\mathrm{jr}} \overrightarrow{(\mathrm{k})}$ is a basis function that transforms as the $r^{\text {th }}$ column of the $j^{\text {th }}$ irreducible representation, and is a linear combination of spherical harmonics of order $l$. This discussion is sufficient to prove theorem III.

Theorem III: A function $\mathrm{y}_{\mathrm{j}}^{\mathrm{r}}(\overline{\mathrm{k}})$ is a basis function for the group $\mathscr{G}$ if and only if

$$
\mathrm{y}_{\mathrm{j}}^{\mathrm{r}} \overline{(\mathrm{k})}=\sum_{l} \mathrm{~b}_{l} \mathrm{x}_{l}^{\mathrm{jr}} \overline{(\mathrm{k})}
$$


Proof: If equation (B5) is assumed to be true, then operation upon $y_{j}^{r}$ by an element of $\mathscr{G}$ gives

$$
\begin{aligned}
\mathscr{T y}_{\mathrm{j}}^{\mathrm{r}} & =\sum_{l} \mathrm{~b}_{l} \mathfrak{A x} \mathrm{x}_{l}^{\mathrm{jr}} \overline{(\mathrm{k})} \\
& =\sum_{l} \mathrm{~b}_{l} \sum_{\mathrm{s}} \mathrm{x}_{l}^{\mathrm{js}} \overline{(\mathrm{k})} \mathrm{D}_{\mathrm{sr}}^{\mathrm{j}}(\mathrm{R}) \quad \text { by equation (B2) } \\
& =\sum_{\mathrm{s}} \sum_{l} \mathrm{~b}_{l} \mathrm{x}_{l}^{\mathrm{jr}} \overline{(\mathrm{k}) \mathrm{D}_{\mathrm{sr}}^{\mathrm{j}}}(\mathrm{R})
\end{aligned}
$$

or, finally,

$$
\mathscr{A} \mathrm{y}_{\mathrm{j}}^{\mathrm{r}}=\sum_{\mathrm{s}} \mathrm{y}_{\mathrm{j}}^{\mathrm{s}} \overline{(\mathrm{k})} \mathrm{D}_{\mathrm{sr}}^{\mathrm{j}}(\mathrm{R})
$$

as desired. As usual, the "if" part of the proof is more involved.

Since the $\mathrm{X}_{l}^{\mathrm{j} r}$ form a complete set of functions, the most general expansion for a function belonging to the $\mathrm{j}^{\text {th }}$ irreducible representation of $\mathscr{G}$ is

$$
\mathrm{y}_{\mathrm{j}}^{\mathrm{r}} \overline{(\mathrm{k})}=\sum_{l, \mathrm{~s}} \mathrm{~b}_{l}^{\mathrm{rs}} \mathrm{x}_{l}^{\mathrm{j} \mathrm{s}} \overline{(\mathrm{k})}
$$

The part of theorem III that is to be proven is that the expansion coefficients $b$ are independent of the column indices $r$ and $s$. This expansion, when substituted into equation (B2), gives

$$
\mathscr{A} \mathrm{y}_{\mathrm{j}}^{\mathrm{r}}(\overline{\mathrm{k}})=\sum_{l, \mathrm{~s}} \mathrm{~b}_{l}^{\mathrm{rs}} \mathrm{x}_{l}^{\mathrm{js}}(\overline{\mathrm{k}})=\sum_{l, \mathrm{~s}} \mathrm{~b}_{l}^{\mathrm{rs}} \sum_{\mathrm{p}} \mathrm{x}_{l}^{\mathrm{jp}}(\overline{\mathrm{k}}) \mathrm{D}_{\mathrm{ps}}^{\mathrm{j}}(\mathrm{R})
$$

and

$$
\sum_{\mathrm{r}, \mathrm{s}} \mathrm{y}_{l}^{\mathrm{s}} \overline{(\mathrm{k}) \mathrm{D}_{\mathrm{sr}}^{\mathrm{j}}}(\mathrm{R})=\sum_{\mathrm{s}} \sum_{l, \mathrm{q}} \mathrm{b}_{l}^{\mathrm{sq}} \mathrm{X}_{l}^{\mathrm{jq}}(\overline{\mathrm{k}}) \mathrm{D}_{\mathrm{sr}}^{\mathrm{j}}(\mathrm{R})
$$


Equation (B2) states that these two forms must be identical. Since the $\mathrm{X}_{l}^{\mathrm{Jr}}$ are orthonormal, a multiplication by an $\mathrm{x}_{l}^{\mathrm{jr} *}$ and an integration will reduce both forms, and the result is

$$
\sum_{s} b_{l}^{r s} D_{p s}^{j}(R)=\sum_{s} b_{l}^{s p_{D}^{j}}{ }_{s r}^{(R)}
$$

for each $\mathrm{j}, l, \mathrm{r}$, and $\mathrm{p}$. The pertinent result from group theory is an orthogonality relation for the matrices of irreducible representations

$$
\sum_{p} D_{r p}^{j}(R) D_{s p}^{j}(R)=\delta_{r s}
$$

which is simply the statement of unitarity of the irreducible representations. Using this result then,

$$
\mathrm{b}_{l}^{\mathrm{rr}}=\sum_{\mathrm{S}} \mathrm{b}_{l}^{\mathrm{ss}} \sum_{\mathrm{p}} \mathrm{D}_{\mathrm{sr}}^{\mathrm{j}}(\mathrm{R}) \mathrm{D}_{\mathrm{pr}}^{\mathrm{j}}(\mathrm{R})
$$

The final result is obtained from a second orthogonality relation

$$
\sum_{R} D_{r s}^{j}(R) D_{p q}^{j}(R)=\frac{g}{n_{j}} \delta_{r p} \delta s q
$$

where the dimension of the $j^{\text {th }}$ representation is again $n_{j}$. Thus, summing over all elements of the group in equation (B7) and applying equation (B8) give

$$
\mathrm{gb}_{l}^{\mathrm{rr}}=\frac{\mathrm{g}}{\mathrm{n}_{\mathrm{j}}} \sum_{\mathrm{s}} \mathrm{b}_{l}^{\mathrm{ss}}
$$

for all $r$. This means that the $b_{l}$ are independent of $r$, and completes the proof of theorem III.

Theorem II (p. 23) is a statement about the form of the expansion of a kernel function in terms of the symmetry-adapted functions and, by extension, about its eigenvalues and eigenfunctions. 
Theorem $\Pi$ : Given a function $\mathrm{Q}\left(\overline{\mathrm{k}}, \overline{\mathrm{k}}^{\prime}\right)$ that is invariant under all operations $\mathscr{R}$ of a group $\mathscr{G}$ (i. e., $\mathrm{Q}\left(\overline{\mathrm{k}}, \overline{\mathrm{k}}^{\prime}\right)=\mathrm{Q}\left(\mathrm{R} \overline{\mathrm{k}}, \mathrm{R} \overline{\mathrm{k}}^{\prime}\right)$ for all $\mathscr{R}$ in $\left.\mathscr{G}\right)$, then there exists the expansion

$$
\mathrm{Q}\left(\overline{\mathrm{k}}, \overline{\mathrm{k}}^{\prime}\right)=\sum_{\substack{l, l^{\prime} \\ \mathrm{j}}} \mathrm{A}_{l l^{\prime}}^{\mathrm{j}} \sum_{\mathbf{r}} \mathrm{X}_{l}^{\mathrm{jr}} \overline{(\mathrm{k})} \mathrm{x}_{l^{\prime}}^{\mathrm{jr} *} \overline{\left(\mathrm{k}^{\prime}\right)}
$$

Proof: The function $\mathrm{Q}\left(\overline{\mathrm{k}}, \overline{\mathrm{k}}^{\prime}\right)$ considered successively as a function of $\overline{\mathrm{k}}$ and a function of $\overline{\mathrm{k}^{\prime}}$ certainly possesses the general expansion

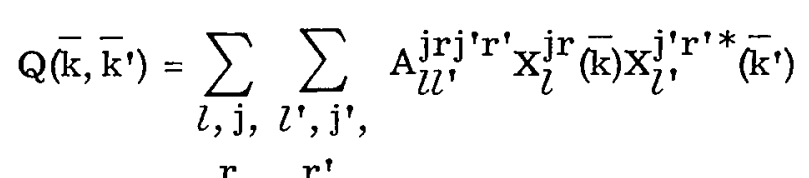

But $\mathrm{Q}\left(\mathrm{R} \overline{\mathrm{k}}, \mathrm{Rk}^{\prime}\right)$ must have the form, from equations (B1) and (B2),

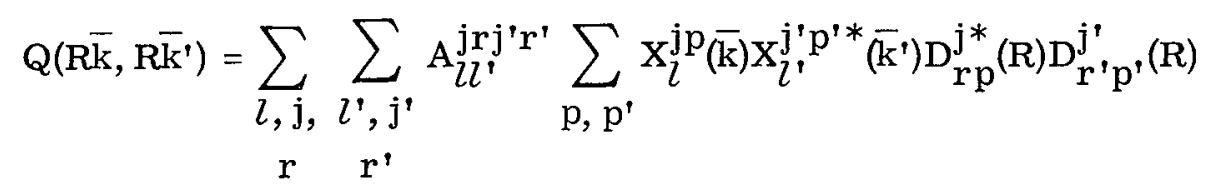

The last two forms must be equal; and using the orthonormality of the symmetryadapted functions they result in

$$
A_{l l^{\prime}}^{j \mathrm{j} j^{\prime} r^{\prime}}=\sum_{p, p^{\prime}} A_{l l^{\prime}}^{j j^{\prime} p^{\prime}} D_{r p}^{j^{*} *}(R) D_{r^{\prime} p^{\prime}}^{j^{\prime}}(R)
$$

A summation over the group elements and the second orthogonality relation (eq. (B8)) yields

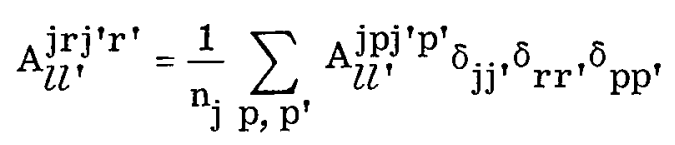

The $\delta$-functions may be used to remove the extraneous indices, and then

$$
\mathrm{A}_{\imath l^{\prime}}^{\mathrm{jr}}=\frac{1}{\mathrm{n}_{\mathrm{j}}} \sum_{\mathrm{p}} \mathrm{A}_{\imath l^{\prime}}^{\mathrm{jp}}
$$


for all $r$. As in the case of the proof of theorem III, it is clear that $A_{l l}^{j}$, cannot depend upon the column index $r$, and then the final result is the statement

$$
Q\left(\overline{\mathrm{k}}, \overline{\mathrm{k}^{\prime}}\right)=\sum_{\substack{l, l^{\prime}, \mathbf{j}}} \mathrm{A}_{l l^{\prime}}^{\mathrm{j}} \sum_{\mathbf{r}} \mathrm{X}_{l^{\mathrm{j}} \mathrm{r}} \overline{(\mathrm{k})} \mathrm{X}_{l^{\prime}}^{\mathrm{j} \mathrm{r}^{*}} \overline{\left(\mathrm{k}^{\prime}\right)}
$$

that was to be proven.

The expansion for the kernel $Q\left(\bar{k}, \bar{k}^{\prime}\right)$ may be integrated to obtain the function $q(\bar{k})$. For the case of a spherical Fermi surface, in which the factor of the magnitude of the Fermi velocity in $d \Omega$ is a constant and may be removed from the integral, the orthogonality of the symmetry-adapted functions leaves only the $l=0$ term for $l^{\prime}$, that is,

$$
q(\bar{k})=\sum_{l} \mathrm{~A}_{20}{ }_{1} \mathrm{x}_{l}{ }^{{ }_{1}} \overline{(\mathrm{k})}
$$

For a nonspherical surface, the magnitude of the Fermi velocity is not a constant, although it is a scalar. Thus, the integral over $\overline{\mathrm{k}}^{\prime}$ involves an integral of $\mathrm{x}_{l}^{\mathrm{jr}}$, over a linear combination of scalar symmetry-adapted functions, and the result is clearly still a scalar in this case. 


\section{REFERENCES}

1. Dresden, M. : Recent Developments in the Quantum Theory of Transport and Galvanomagnetic Phenomena. Rev. Mod. Phys., vol. 33, no. 2, Apr. 1961, pp. 265-342.

2. Ziman, J. M.: Electrons and Phonons. Clarendon Press, Oxford, 1960.

3. Pippard, A. B. : Experimental Analysis of the Electronic Structure of Metals. Reports on Progress in Physics. Vol. 23. A. C. Stickland, ed., The Physical Society 1960 , pp. 176-266.

4. Harrison, W. A.; and Webb, M. B., eds.: The Fermi Surface. John Wiley \& Sons, Inc. , 1960.

5. Mott, N. F.; and Jones, H.: The Theory of the Properties of Metals and Alloys. Clarendon Press, Oxford, 1936; reprinted by Dover Publications, Inc., 1958.

6. Wannier, Gregory H.: Elements of Solid State Theory. Cambridge University Press, 1959.

7. Wilson, Alan H.: The Theory of Metals. Second ed., Cambridge University Press, 1953.

8. Taylor, P. L.: The Boltzmann Equation for Conduction Electrons. Proc. Roy. Soc. (London), ser. A, vol. 275, no. 1361, Sept. 24, 1963, pp. 200-208.

9. Taylor, P. L.: The Relaxation Time of Conduction Electrons in the Noble Metals. Proc. Roy. Soc. (London), ser. A, vol. 275, no. 1361, Sept. 24, 1963, pp. 209-217.

10. Roman, Paul: Advanced Quantum Theory. Addison-Wesley Publ. Co., Inc., 1965.

11. Harrison, Walter A.: Pseudopotentials in the Theory of Metals. W. A. Benjamin, Inc. , 1966.

12. Chambers, R. G.: The Kinetic Formulation of Conduction Problems. Proc. Phys. Soc. (London), ser. A, vol. 65, pt. 6, June 1952, pp. 458-459.

13. Budd, Herbert: Chambers' Solution of the Boltzmann Equation. Phys. Rev., vol. 127, no. 1, July 1, 1962, p. 4.

14. Budd, Herbert: Contributions a la Theorie des Phenomenes de Transport. Publications Scientifiques et Techniques No. 408, Ministere de l'Air, Paris, 1964.

15. Cohen, M. H.; Harrison, M. J.; and Harrison, W. A.: Magnetic-Field Dependence of the Ultrasonic Attenuation in Metals. Phys. Rev., vol. 117, no. 4, Feb. 15, 1960, pp. 937-952. 
16. Kittel, Charles: Quantum Theory of Solids. John Wiley \& Sons, Inc., 1963.

17. Smith, Arthur C.; Janak, James F.; and Adler, Richard B.: Electronic Conduction in Solids. McGraw-Hill Book Co., Inc., 1967.

18. Lifshitz, I. M. ; Azbel', M. Ia. ; and Kaganov, M. I. : The Theory of Galvanomagnetic Effects in Metals. Soviet Phys.-JETP, vol. 4, no. 1, Feb. 1957, pp. 41-54.

19. Lifshitz, I. M. ; and Peschanskii, V. G. : Galvanomagnetic Characteristics of Metals with Open Fermi Surfaces, I. Soviet Phys.-JETP, vol. 8, no. 5, May 1959, pp. 875-883.

20. Lifshitz, I. M.; and Peschanskii, V. G. : Galvanomagnetic Characteristics of Metals with Open Fermi Surfaces, II. Soviet Phys.-JETP, vol. 11, no. 1, July 1960, pp. 137-141.

21. Sondheimer, E. H.: The Boltzmann Equation for Anisotropic Metals. Proc. Roy. Soc. (London), ser. A, vol. 268, no. 1332, June 19, 1962, pp. 100-108.

22. Mikhlin, Solomon G. : Integral Equations and Their Applications to Certain Problems in Mechanics, Mathematicial Physics and Technology. Second ed., Macmillan Co., 1964.

23. Courant, R.; and Hilbert, D.: Methods of Mathematical Physics. Vol. I. Interscience Publ., Inc., 1953.

24. Jones, M. C. ; and Sondheimer, E. H. : Magnetoresistance Anisotropy in Metals Due to Anisotropic Scattering of Electrons. Phys. Rev., vol, 155, no. 3, Mar. 15, 1967, pp. 567-576.

25. Mann, E. : Energieänderung der Leitungselektronen durch Punktfehler in Metallen. I. Allgemeine Theorie. Phys. Status Solidi, vol. 11, 1965, pp. 753-766.

26. Mann, E. : Energieänderung der Leitungselektronen durch Punktfehler in Metallen. II. Beispiele und Anwendungen. Phys. Status Solidi, vol. 11, 1965, pp. 767-778.

27. Mann, E. : Streuung der Leitungselektronen durch Punktfehler in Metallen. Phys. Status Solidi, vol. 13, 1966, pp. 293-306.

28. Migdal, A. B.: The Momentum Distribution of Interacting Fermi Particles. Soviet Phys.-JETP, vol. 5, no. 2, Sept. 1957, pp. 333-334.

29. Abrikosov, A. A.; Gorkov, L. P.; and Dzyaloshinski, I. E. (Richard A. Silverman, trans. ): Methods of Quantum Field Theory in Statistical Physics. Prentice-Hall, Inc. , 1963.

30. Prigogine, I. : Non-Equilibrium Statistical Mechanics. Interscience Publ., Inc. , 1962. 
31. Edwards, S. F.: A New Method for the Evaluation of Electric Conductivity in Metals. Phil. Mag., vol. 3, no. 33, Sept. 1958, pp. 1020-1031.

32. Fair, Gale; and Taylor, P. L. : Exact Semiclassical Theory for Galvanomagnetic Effects in Metals. NASA TN D-4166, 1967.

33. Riesz, F.; and Sz.-Nagy, B.: Functional Analysis. Frederick Ungar Publ. Co., 1955.

34. Wigner, E. P. (J. J. Griffin, trans.): Group Theory and Its Application to the Quantum Mechanics of Atomic Spectra. Academic Press, 1959.

35. Melvin, M. A.: Simplification in Finding Symmetry-Adapted Eigenfunctions. Rev. Mod. Phys., vol. 28, no. 1, Jan. 1956, pp. 18-44.

36. Von der Lage, F. C.; and Bethe, H. A.: A Method for Obtaining Electronic Eigenfunctions and Eigenvalues in Solids with an Application to Sodium. Phys. Rev., vol. 71, no. 9, May 1, 1947, pp. 612-622.

37. Altmann, S. L.; and Cracknell, A. P.: Lattice Harmonics I. Cubic Groups. Rev. Mod. Phys., vol. 37, no. 1, Jan. 1965, pp. 19-32.

38. Bouchaert, L.; Smoluchowski, R.; and Wigner, E. P. : Theory of Brillouin Zones and Symmetry Properties of Wave Functions in Crystals. Phys. Rev., vol. 50, no. 1, July 1, 1936, pp. 58-67.

39. Hamermesh, Morton: Group Theory and Its Applications to Physical Problems. Addison-Wesley Publ. Co., Inc., 1962.

40. Klauder, J. R.; Reed, W. A.; Brennert, G. F.; and Kunzler, J. E. : Study of the Fine Structure in the High-Field Galvanomagnetic Properties and the Fermi Surface of Copper. Phys. Rev., vol. 141, no. 2, Jan. 1966, pp. 592-603.

41. Francis, J. G. F.: The QR Transformation: A Unitary Analogue to the LR Transformation. I. Computer J., vol. 4, Oct. 1961, pp. 265-271.

42. Francis, J. G. F.: The QR Transformation. II. Computer J., vol. 4, 1962, pp. $332-345$.

43. Wilkinson, J. H.: The Algebraic Eigenvalue Problem. Clarendon Press, Oxford, 1965.

44. Babiskin, J.; and Siebenmann, P. G.: Saturating and Linear Magnetoresistive Effects in Sodium and Potassium. Phys. kondens. Materie, vol. 9, no. 1/2, Mar. 10, 1969, pp. 113-121. 
45. Seeger, A.; Mann, E.; and Clausecker, K.: Magnetoresistivity of Metals, I. General Theory. Phys. Status Solidi, vol. 24, 1967, pp. 721-732.

46. Stone, M. H. : Linear Transformations in Hilbert Space. American Math. Soc. Colloquium Publ. Vol. 15, 1932. 


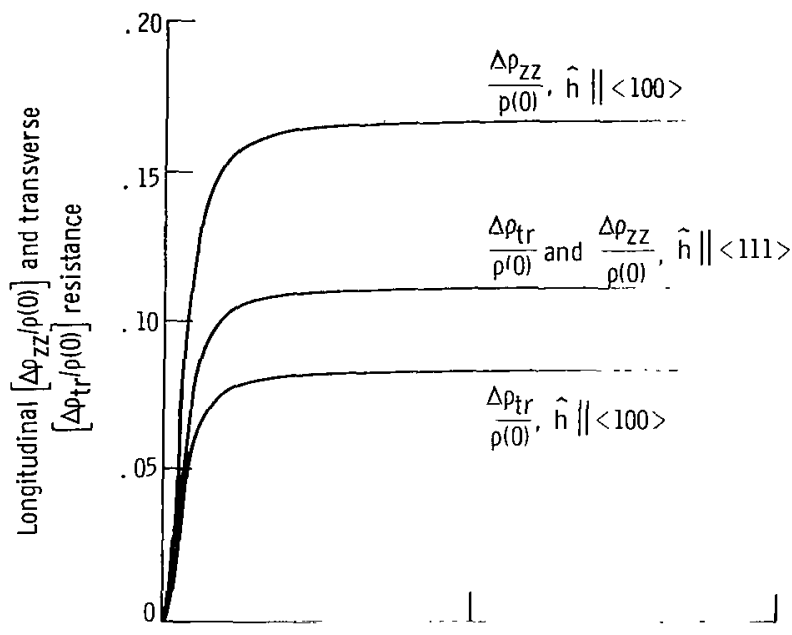

(a) Magnetoresistance for two symmetry directions, $\langle 100\rangle$ and $\langle 111\rangle$.

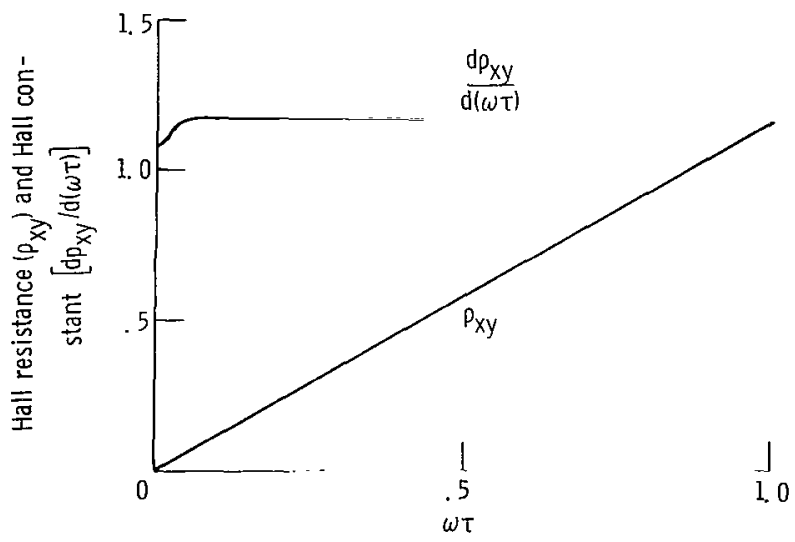

(b) Hall effect for symmetry direction $\langle 111\rangle$.

Figure 1. - Magnetoresistance and Hall effect for weakscattering case, $A_{13}=0.06275$. 


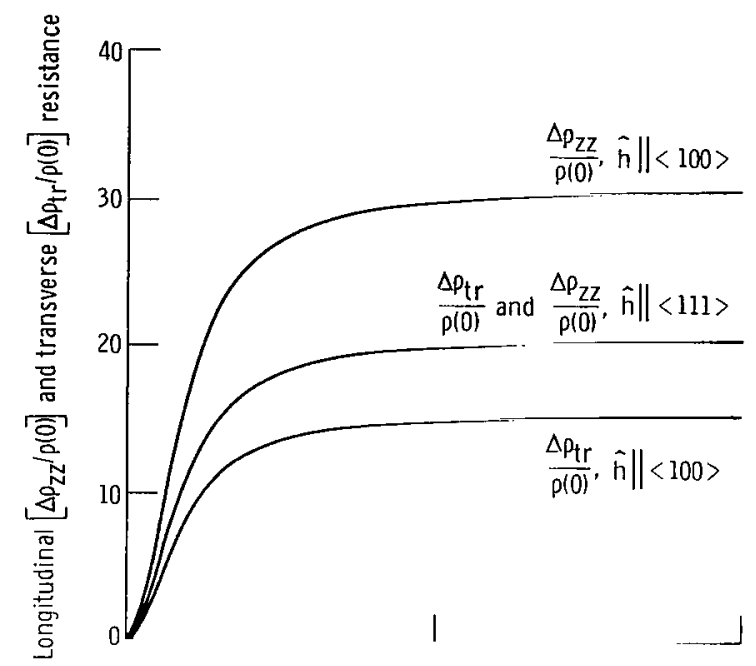

(a) Magnetoresistance for two symmetry directions, $\langle 100\rangle$ and $\langle 111\rangle$.

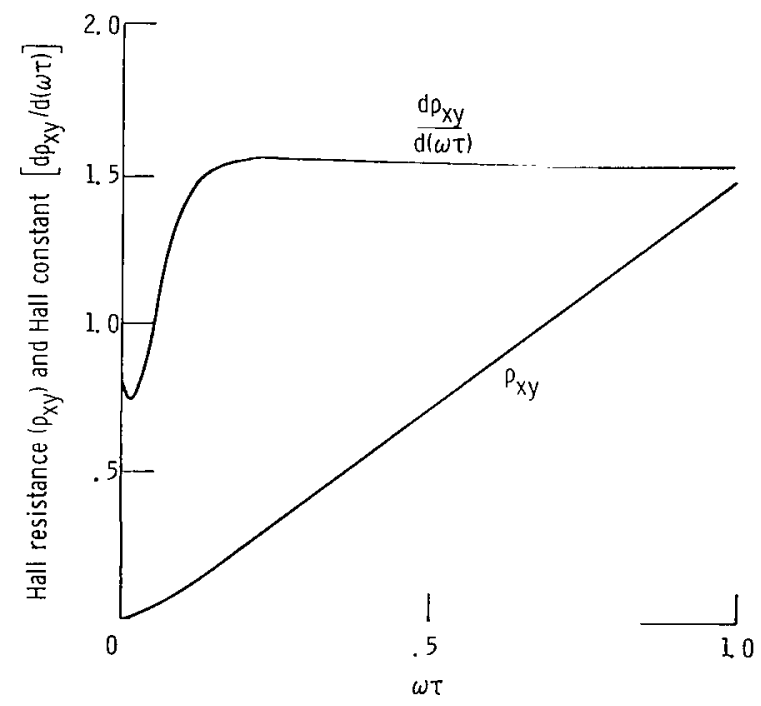

(b) Hall effect for symmetry direction $\langle 111\rangle$.

Figure 2. - Magnetoresistance and Hall effect for strongscattering case, $A_{13}=0.26726$. 


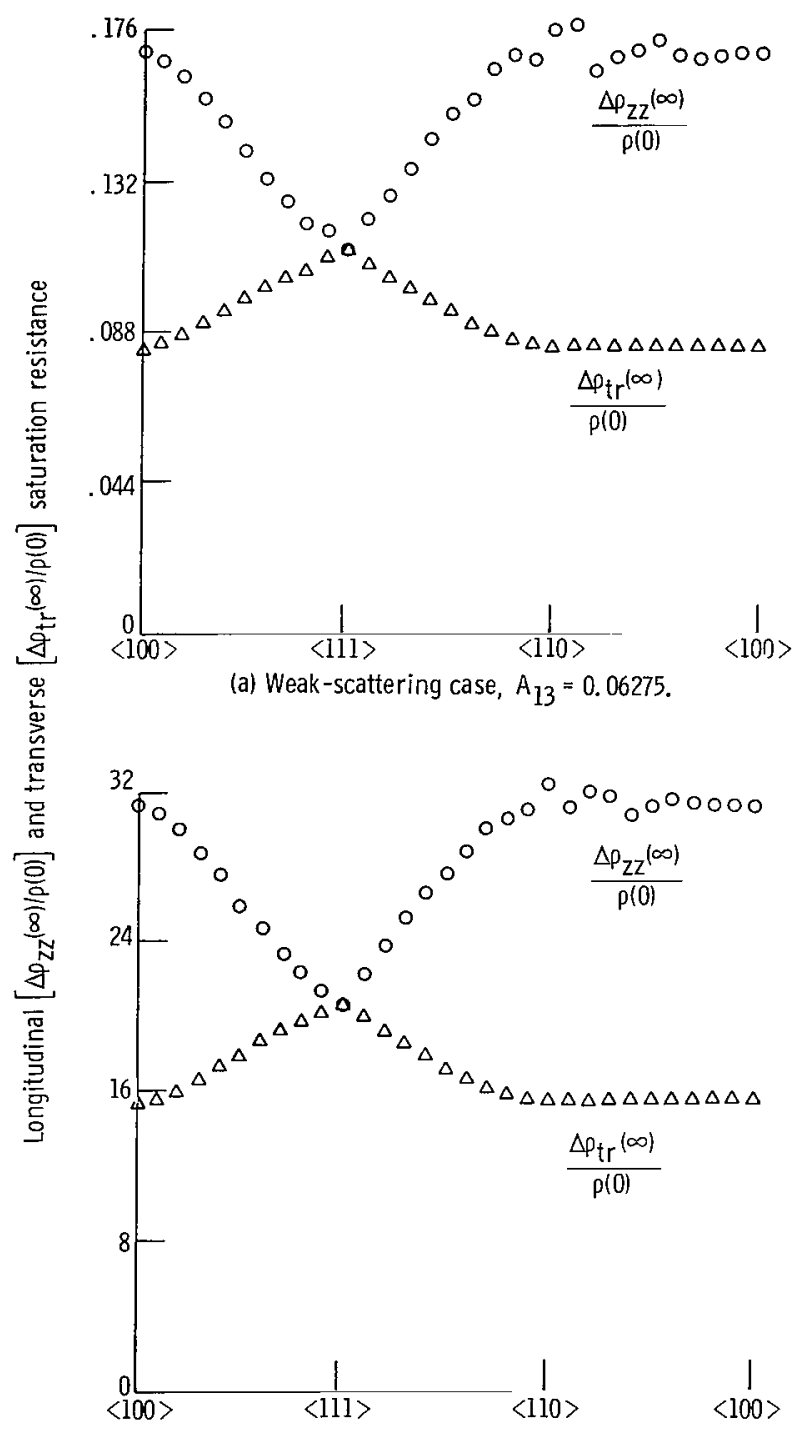

(b) Strong-scattering case, $A_{13}=0.26726$.

Figure 3. - Saturation magnetoresistance for weak- and strong-scattering cases. 


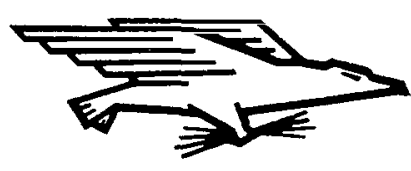

POSTAGE AND FEES PAID NATIONAL AERONAUTICS AND SPACE ADMINISTRATION

$$
\begin{aligned}
& 64000151 \text { ob 305 60328 00903 } \\
& \text { AL FETEF WAYLAS LAELHATORY/WLIL/ }
\end{aligned}
$$

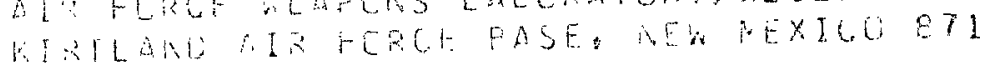

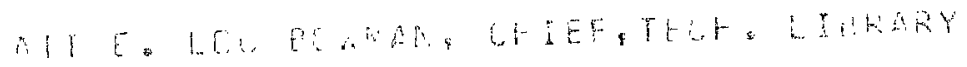

POSTMASTER: $\quad$ If Undeliverable (Section 158

"The acronatical and space activities of the United States shall be conducted so as to contribute. . . to the expansion of human knowledge of phenomena in the atmospbere and space. The Administration sball provide for the widest practicable and appropriate dissemination of information concerning its activities and the results thereof."

- National Aeronautics and Space ACt of 1958

\section{'NASA' SCIENTIFIC AND TECHNICAL PUBLICATIONS}

TECHNICAL REPORTS: Scientific and technical information considered important, complete, and a lasting contribution to existing knowledge.

TECHNICAL NOTES: Information less broad in scope but nevertheless of importance as a contribution to existing knowledge.

TECHNICAL MEMORANDUMS:

Information receiving limited distribution

- because of preliminary data, security classification, or other reasons.

CONTRACTOR REPORTS: Scientific and technical information generated under a NASA contract or grant and considered an important contribution to existing knowledge.
TECHNICAL TRANSLATIONS: Information published in a foreign language considered to merit NASA distribution in English.

SPECIAL PUBLICATIONS: Information derived from or of value to NASA activities. Publications include conference proceedings, monographs, data compilations, handbooks, sourcebooks, and special bibliographies.

TECHNOLOGY UTILIZATION PUBLICATIONS: Information on technology used by NASA that may be of particular interest in commercial and other non-aerospace applications. Publications include Tech Briefs, Technology Utilization Reports and Notes, and Technology Surveys.

Details on the availability of these publications may be obtained from:

\section{SCIENTIFIC AND TECHNICAL INFORMATION DIVISION NATIONAL AERONAUTICS AND SPACE ADMINISTRATION Washington, D.C. 20546}

